\title{
45. ROCK-MAGNETIC STRATIGRAPHY OF SITE 645 (BAFFIN BAY) FROM ODP LEG $105^{1}$
}

\author{
Frank R. Hall ${ }^{2}$ and John W. King ${ }^{2}$
}

\begin{abstract}
We present the rock-magnetic stratigraphy for Ocean Drilling Program (ODP) Leg 105, Site 645 (Baffin Bay). Variations in magnetic mineral concentration $(X)$ and particle size $\left(X_{\mathrm{ARM}} / X\right)$ are used to correlate advanced piston corer (APC) cores from several holes at Site 645. Downsite variations in rock-magnetic parameters and carbonate content are placed within the framework of inferred changes in sedimentation. These results suggest an initiation of Northern Hemisphere ice rafting in Baffin Bay in the early-late Pliocene (approximately 3.4 Ma).
\end{abstract}

\section{INTRODUCTION}

Rock magnetism is the study of the physical properties of magnetic minerals in rocks. We define magnetic minerals as those capable of maintaining remanence at room temperature. The composition and concentration of magnetic minerals within a sediment are often directly related to source area and site of deposition.

Variations in rock-magnetic parameters can be related to environmental changes (Thompson et al., 1980; Thompson and Oldfield, 1986). Positive correlations between rock-magnetic and oxygen-isotope variations within Quaternary deep-sea sediments were reported by King (1986) for the Gulf of Mexico, by Robinson (1986) from the North Atlantic, and by Bloemendal et al. (1988) in the eastern equatorial Atlantic. The rock-magnetic results of Bloemendal et al. also correspond to variations in river influx and eolian dust production. Doh et al. (1988) correlated magnetic mineral parameters, plotted as accumulation rates, with major paleoceanographic events during the last 70 m.y. in their Pacific Core LLGPC-3.

Rock-magnetic studies have the advantage of allowing one to analyze rapidly a large number of samples with equipment that is readily available, relatively inexpensive, and often portable. In addition, rock-magnetic analyses do not physically harm the samples.

Here, we present the results of rock-magnetic analyses performed on sediment samples from Site 645 (Baffin Bay; Fig. 1).

\section{Rock-Magnetic Parameters}

A detailed discussion of rock magnetism is beyond the scope of this paper. To assist the reader, we present a brief discussion of the rock-magnetic parameters used here and recommend more detailed accounts found in other texts (e.g., O'Reilly, 1984; Thompson and Oldfield, 1986).

Magnetic mineral assemblages are usually composed of complex mixtures, and detailed studies of magnetic mineral extractions are required to determine their compositions. However, rock-magnetic parameters are useful for determining relative changes in magnetic mineralogy, grain size, and concentration.

Low-field magnetic susceptibility $(X)$ for a magnetic mineral is defined by the equation:

$$
X=J /(H \cdot \text { mass }) \text {, }
$$

\footnotetext{
${ }^{1}$ Srivastava, S. P., Arthur, M., Clement, B., et al., 1989. Proc. ODP, Sci. Results, 105: College Station, TX (Ocean Drilling Program).

2 University of Rhode Island, Graduate School of Oceanography, Narragansett, RI 02882.
}

where $J$ is the induced magnetization of a sample, and $H$ is the intensity of the applied magnetic field. The volume susceptibility, $k$, is determined by replacing mass with volume in Equation $\# 1$. The susceptibility of a sediment sample is primarily a measure of the concentration of magnetic minerals (e.g., Collinson, 1983). The spinel-group (ferrimagnetic) of minerals (e.g., magnetite and titanomagnetite) has susceptibilities that are up to two orders of magnitude stronger than the canted-antiferromagnetic type (e.g., hematite and goethite). Studies of synthetic samples with known concentrations of magnetite and hematite show that hematite must be present in proportions of $80 \%$ to $90 \%$ to influence magnetic properties significantly (King et al., 1982a). For these reasons, ferrimagnetic minerals dominate the magnetic properties of naturally occurring sediments. The presence of diamagnetic, paramagnetic, and canted-antiferromagnetic minerals can influence the measured susceptibility when ferrimagnetic mineral concentration is low.

Superparamagnetic minerals are ultrafine-grained magnetic minerals unable to maintain stable remanence at room temperature. These will contribute to anomalously high susceptibility values and can be detected by examining the frequency dependence of susceptibility (Stephenson, 1971; Bloemendal et al., 1985): the contribution of superparamagnetic minerals decreases with increasing frequency of the applied field. We measured at two frequencies; the ratio of high-frequency susceptibility $\left(X_{\mathrm{hf}}\right)$ to low-frequency susceptibility $\left(X_{\mathrm{hf}}\right)$ defines frequency dependence.

$X_{\mathrm{ARM}} / X$ is the ratio of anhysteretic susceptibility to magnetic susceptibility. Anhysteretic remanence (ARM) results when a magnetic mineral is placed in the presence of a strong, decaying alternating (AC) magnetic field with a biasing direct (DC) magnetic field. Anhysteretic susceptibility $\left(X_{\text {ARM }}\right)$ is the ARM divided by the intensity of the DC magnetic field.

$X_{\mathrm{ARM}} / X$ is an indicator of the relative particle-size variation of magnetic minerals, with higher (lower) values representing finer (coarser) grains (King et al., 1982b; King, 1986). Superparamagnetic minerals will not effect ARM, but can increase $X$, resulting in data that falsely indicate coarse magnetic material.

Hall et al. (this volume) suggest that $\mathrm{ARM} / X$ can be, in some instances, used as an indicator of the relative grain-size variation of both the magnetic and nonmagnetic fraction of sediments.

SIRM (saturation isothermal remanence) is the maximum room-temperature remanence that a mineral can acquire. Lowcoercivity ferrimagnetic minerals are saturated in magnetic fields of less than $0.3 \mathrm{~T}$, whereas high-coercivity canted-antiferromagnetic minerals often require $1 \mathrm{~T}$ or higher.

BIRM (back-isothermal remanence) is an isothermal remanence antiparallel to the SIRM. We apply a BIRM with a mag- 


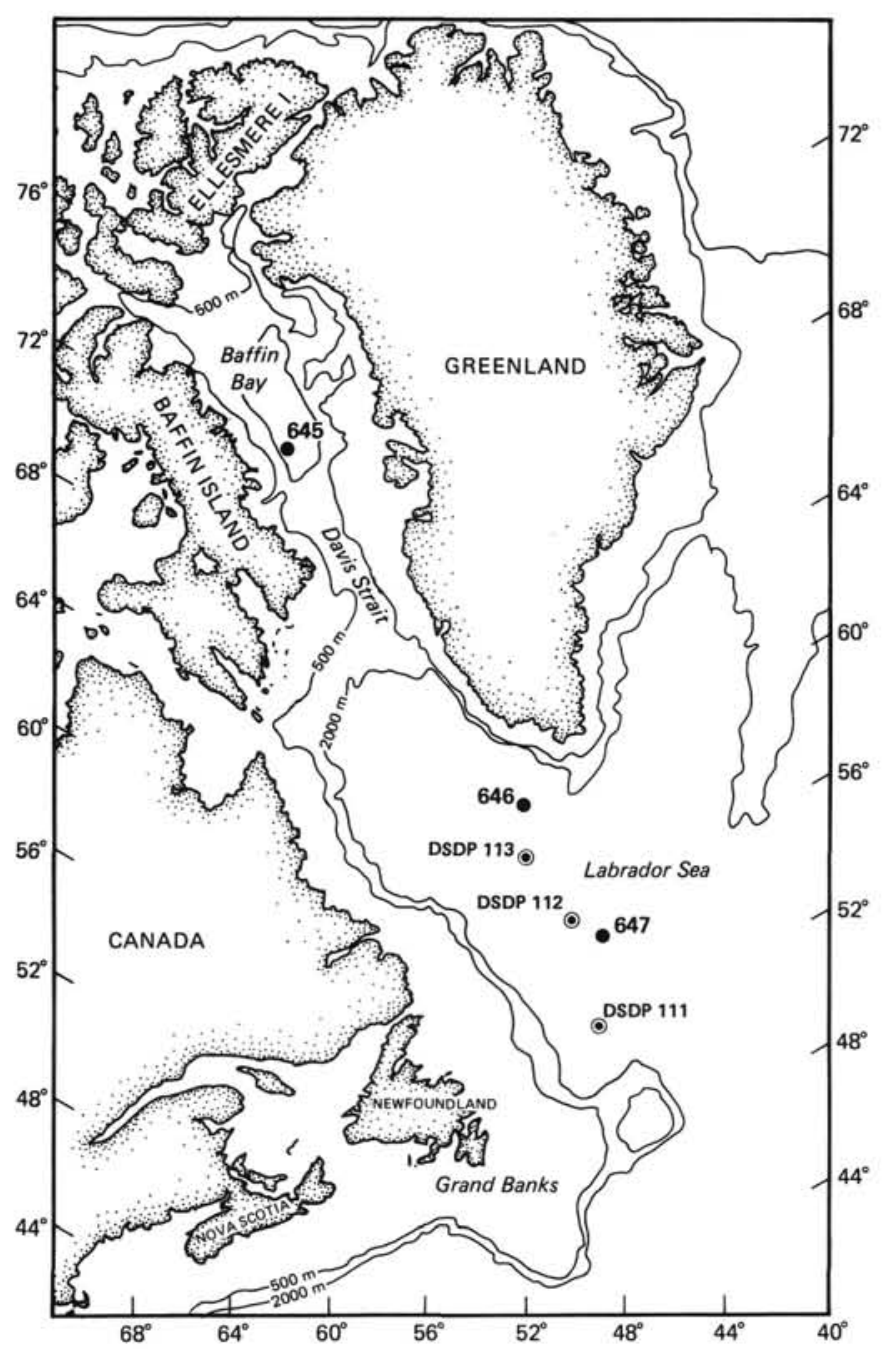

Figure 1. Location of Site 645 (Baffin Bay).

netic field strength that is strong enough to saturate ferrimagnetic minerals, but too weak to saturate canted-antiferrimagnetic minerals.

HIRM is defined by the equation:

$$
\text { HIRM }=(\text { SIRM }+ \text { BIRM }) /(2 \cdot \text { sample mass }),
$$

and is indicative of the relative concentration of canted-antiferromagnetic minerals.

$S$ is defined as

$$
S=\mathrm{BIRM} / \mathrm{SIRM}
$$

and expresses the concentration of canted-antiferromagnetic minerals with respect to ferrimagnetic minerals.

If the magnetic mineral assemblage was composed of only ferrimagnetic minerals, then we would expect HIRM values near zero, and $S$ values near one. Deviations from these values are attributed to the presence of canted-antiferromagnetic minerals.

Units for rock-magnetic analyses used here are given in the Appendix.

\section{METHODS}

Samples were collected during Leg 105 and on shore. A square chimney was inserted into the sediment, and a sample exhumed from the core. The bottom of the sample adjacent to the core liner was removed because of the possibility of sediment disturbance. The samples were placed in $5-\mathrm{cm}^{3}$ cubes, stored in plastic containers, and refrigerated to minimize desiccation. Samples were weighed, including the plastic cubes, using an electronic balance accurate to $0.01 \mathrm{~g}$. A total of $3 \mathrm{~g}$, the average of 100 cubes with ODP sample labels attached, was subtracted from each sample.

Low-field magnetic susceptibility $(X)$ was measured on each archive half of the APC cores using a Bartington whole-core susceptibility sensor during Leg 105, to the base of Chronozone C1N. In addition, the susceptibilities of all discrete samples were measured using a Bartington Instruments dual-frequency magnetic susceptibility meter (low frequency $=0.47 \mathrm{kHz}$, high frequency $=4.7 \mathrm{kHz}$ ). Details of these measurements were discussed in Srivastava, Arthur, et al. (1987).

All samples were demagnetized at $1 \mathrm{~T}$ using a Schoenstedt GSD-1 alternating-field demagnetizer before application of an ARM. We used a peak AC field intensity of $100 \mathrm{mT}$ and a DC field of $0.05 \mathrm{mT}$ (Site 645 APC core) or $0.1 \mathrm{mT}$ (downhole Site 645).

SIRM and BIRM were applied at $1 \mathrm{~T}$ (the limit of the DC electromagnet) and $-0.3 \mathrm{~T}$, respectively. Both $\mathrm{ARM}_{0.1} \mathrm{~T}$ and IRM measurements were performed using a DIGICO slow-spin fluxgate magnetometer. $\mathrm{ARM}_{\mathrm{O} .05 \mathrm{~T}}$ measurements were performed using the cryogenic magnetometer.

Using the $X_{\mathrm{ARM}} / X$ records of Holes $645 \mathrm{~A}, 645 \mathrm{C}$, and $645 \mathrm{~F}$ and the visual core correlation points in Srivastava, Arthur, et al. (1987, p. 81), nine tie-points were determined (Fig. 1). We used only six tie-points with Hole $645 \mathrm{~A}$ because of evidence of core disturbance at the bottom. These tie-points were put into the CORPAC correlation program of Martinson et al. $(1982,1987)$. Using these tie-points, the CORPAC program allowed us to stack the rock-magnetic records, develop a composite downhole curve for each rock-magnetic parameter, and determine the degree of between-hole correlation for each parameter we studied. With the CORPAC program, we were also able to make Shaw diagrams (plots of equivalent depths of paired cores from a given area). Shaw diagrams were made to relate Holes $645 \mathrm{~A}$ and $645 \mathrm{~F}$ with Hole $645 \mathrm{C}$.

\section{RESULTS AND DISCUSSION}

Shipboard rock-magnetic results of whole-core and discretesample magnetic susceptibilities were discussed in Srivastiva, Arthur, et al. (1987) and thus are not discussed here in detail. Data reported here are primarily from post-cruise sampling. Results are tabulated in the Appendix.

Because of the large number of data points collected from whole-core susceptibility logs, these results are not in the Appendix, but may be obtained by contacting the authors or ODP.

\section{Site 645}

\section{Advanced Piston Cores (APCs)}

In Srivastava, Arthur, et al. (1987), whole-core susceptibilities were used to correlate between holes. In addition, the frequency dependence of susceptibility was shown to be unimportant.

Figures 2 through 5 show rock-magnetic results from discrete samples. Nine tie-points are shown in Figure 2.

Figure 6 shows the composite records of the stacked rockmagnetic data using the same nine tie-points in Figure 2 and other rock-magnetic parameters. These data have good correlation coefficients $(R)$ between holes for $X_{\mathrm{ARM}} / X(R=0.846)$ and $X(R=0.673)$. However, the correlations are poor with respect to HIRM $(R=0.324)$ and $S(R=0.028)$. These data suggest magnetic mineral concentration and grain size may be potentially useful for between-hole correlation in the upper few meters of sediment in Baffin Bay, whereas magnetic mineralogy is not.

Shaw diagrams correlating the depths of Holes 645A and $645 \mathrm{~F}$ with respect to $645 \mathrm{C}$ are shown in Figure 7.

Hole $645 \mathrm{G}$ was sampled below the sediment-water interface at a suggested depth of $1 \mathrm{~m}$ (Srivastava, Arthur, et al., 1987). We cannot correlate this hole with the other APCs using rock- 
$645 \mathrm{~A}$

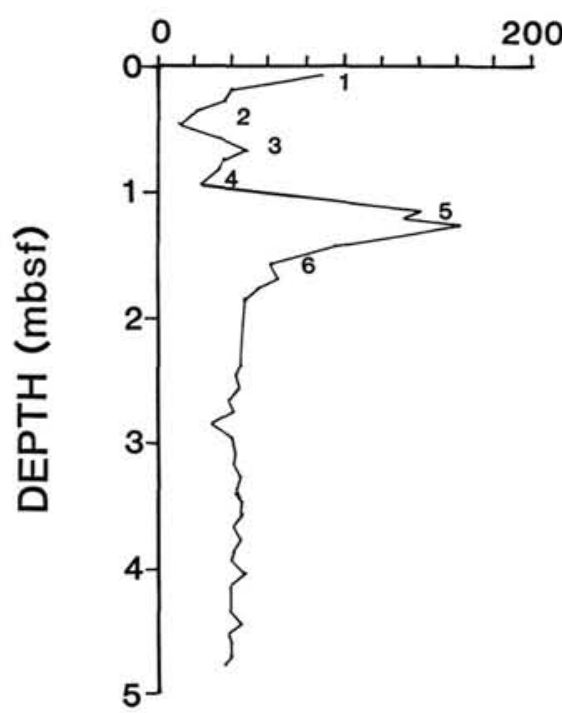

$645 \mathrm{C}$

$645 \mathrm{~F}$

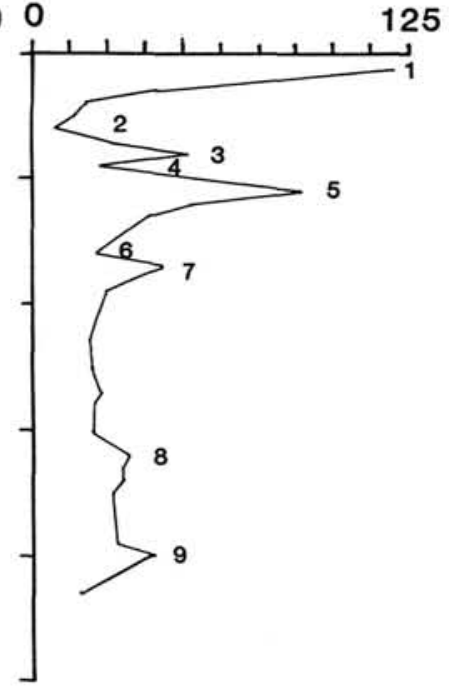

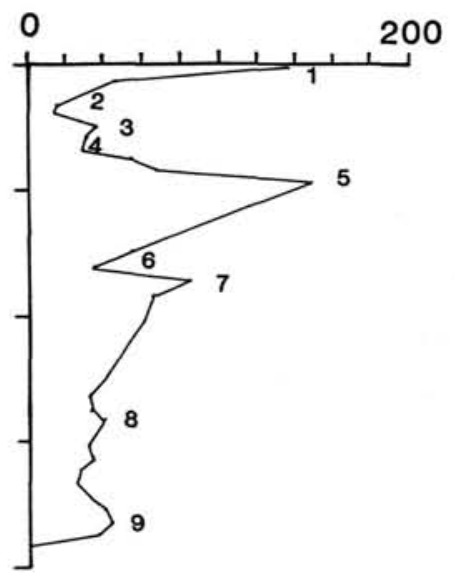

Figure 2. Downhole correlations of $X_{\mathrm{ARM}} / X$ parameters from discrete APC samples from Site 645 . Numbers on the figures are the tie-points.

$645 \mathrm{~A}$

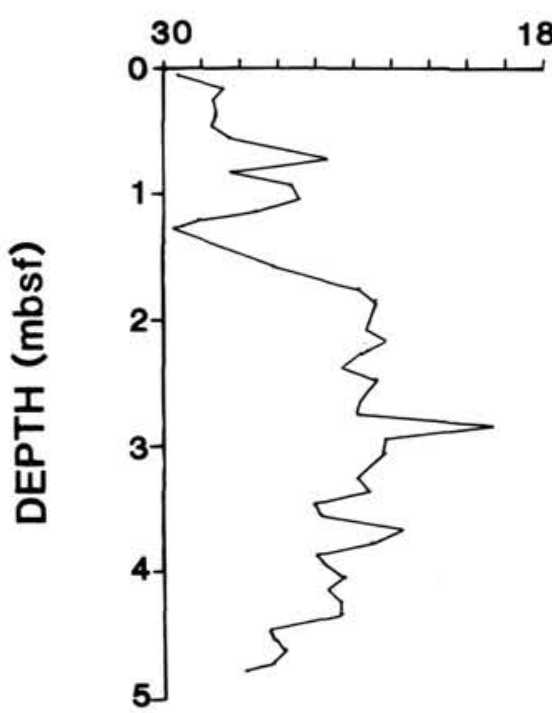

$645 \mathrm{C}$

$645 \mathrm{~F}$

8020

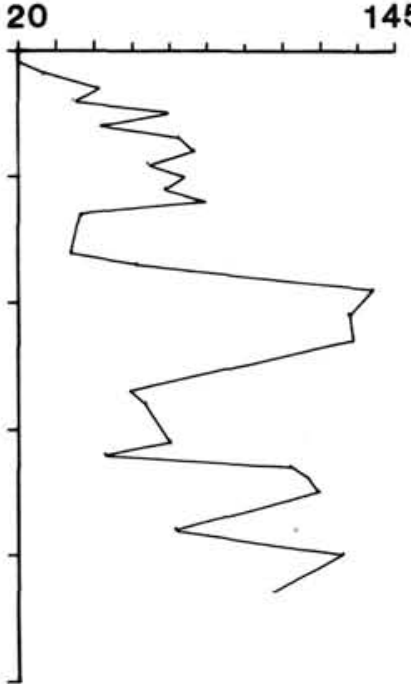

200

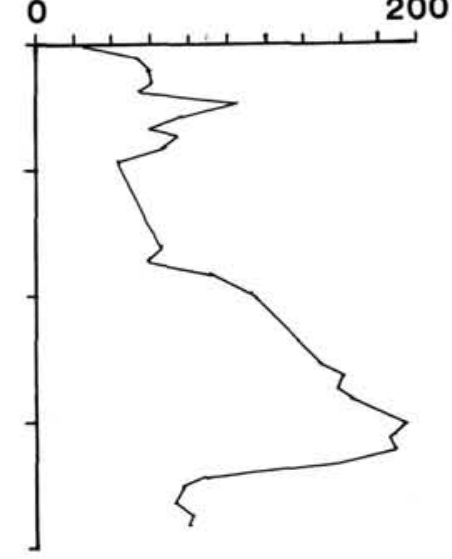

Figure 3. Downhole variations in susceptibility $(X)$. 
645A

$645 C$

$645 F$
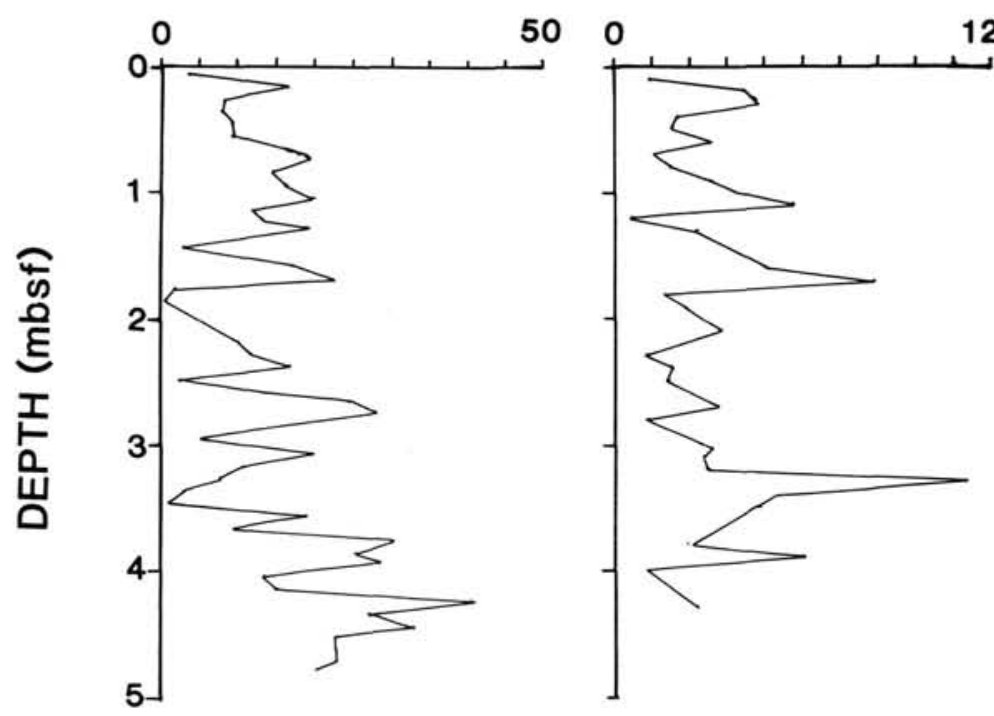

125

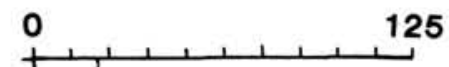

Figure 4. Downhole variations in HIRM.

645A

$645 C$

$645 \mathrm{~F}$

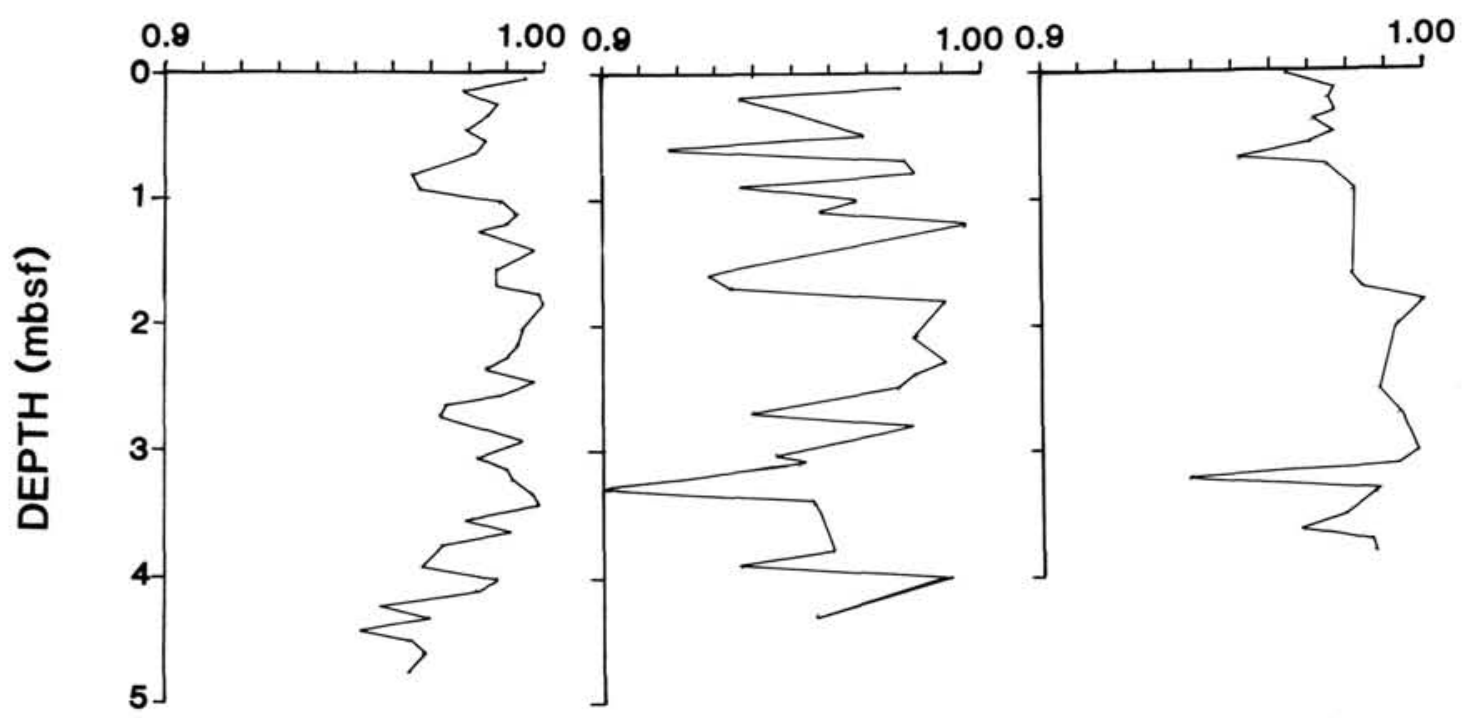

Figure 5. Downhole variations in $S$. 


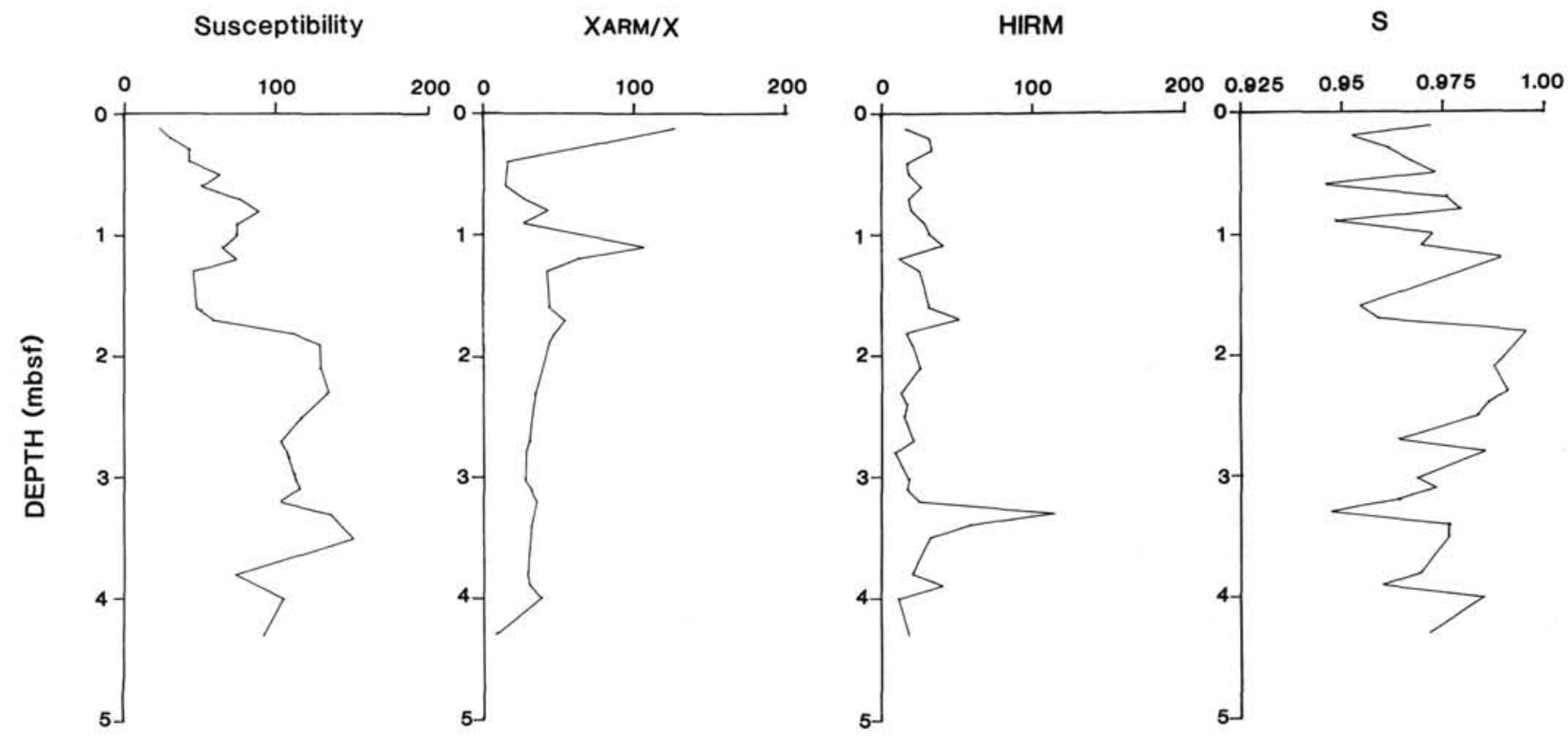

Figure 6. Stacked records of rock-magnetic parameters.

DEPTH (mbsf)

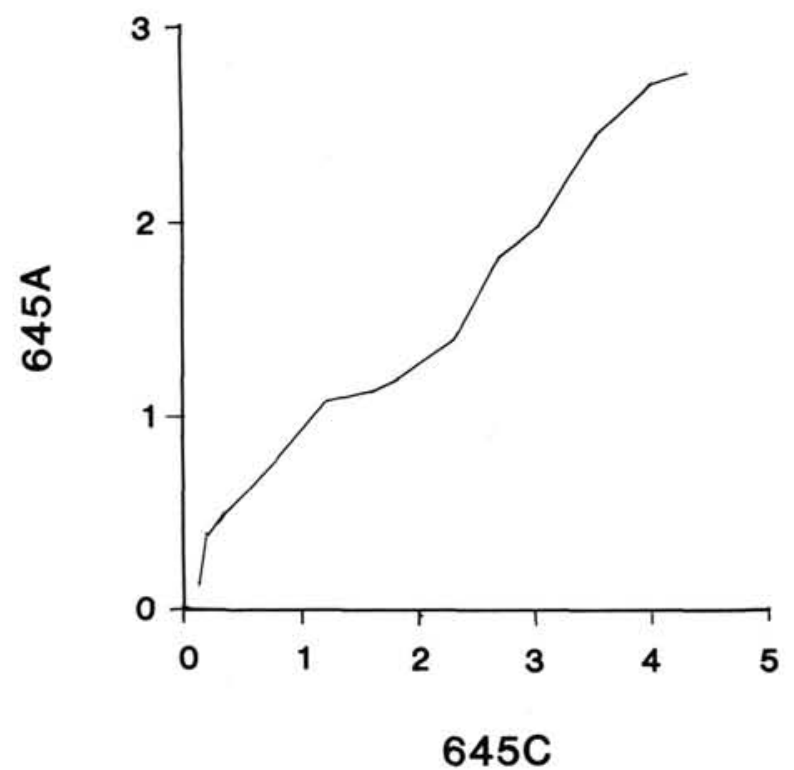

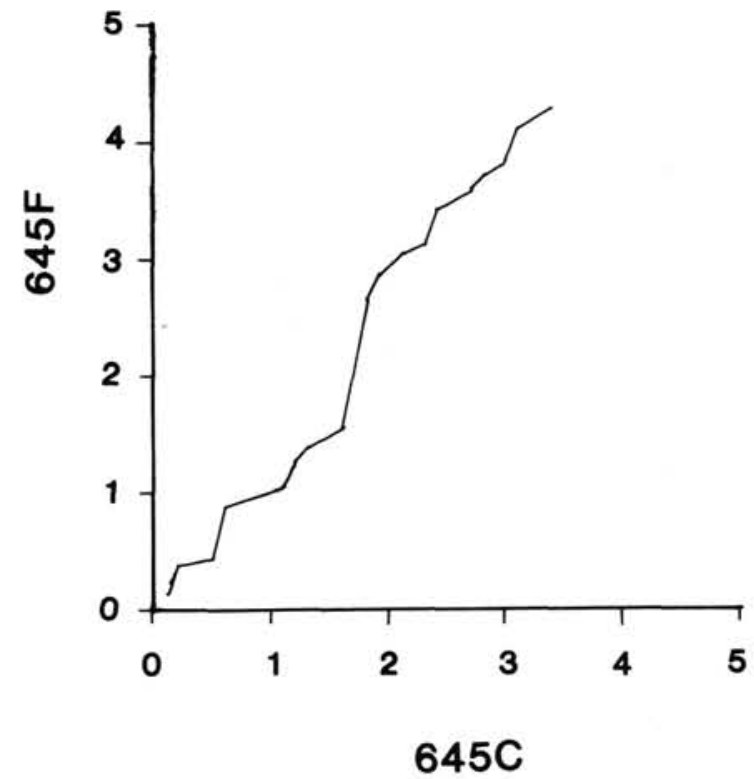

Figure 7. Shaw diagrams for APC cores at Site 645. Depths of tie-points from Figure 1 were used to correlate the cores.

magnetic parameters and did not include Hole 645G in our correlations.

\section{Downhole Variations of Site 645}

The deposition of magnetic minerals in Baffin Bay reflects variations in the mode of transport and source area of sediments. $X, X_{\mathrm{ARM}} / X$, and HIRM show marked differences between pre-glacial (lithologic Unit III: lower-middle Miocene to upper Pliocene) and glacial (lithologic Units I and II: upper Pliocene to Holocene) sequences.
Variations in the rock-magnetic parameter $X$ follow the same trend as $\mathrm{CaCO}_{3}$ downhole (Fig. 8). Previous comparisons of $X$ with $\mathrm{CaCO}_{3}$ in other regions (Robinson, 1986; Bloemendal et al., 1988) have shown an inverse relationship, with $X$ being diluted by pelagic carbonate. However, much of the carbonate in the upper part of Site 645 is detrital (Srivastava, Arthur, et al., 1987). Therefore, variations in carbonate with respect to susceptibility will reflect the source area, not pelagic deposition alone.

Lithologic Unit III has been divided into three subunits: IIIA (335-753.4 mbsf), IIIB (753.4-916.8 mbsf), and IIIC (916.8- 

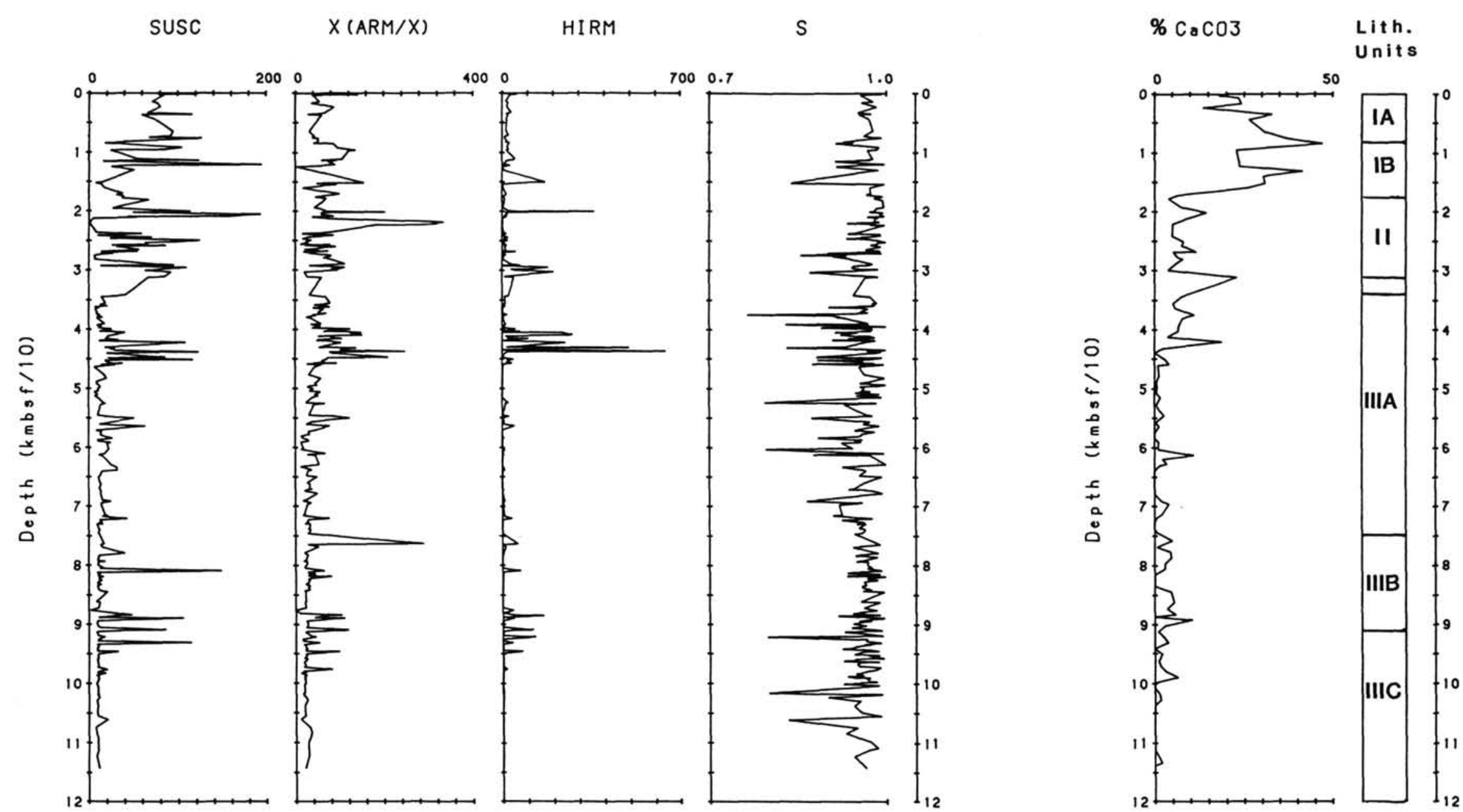

Figure 8. Downsite variations in rock-magnetic parameters and carbonate concentration at Site 645 . The gap between lithologic Units II and III is an area of no recovery. HIRM values over 700 $\mathrm{mA} / \mathrm{m} \cdot \mathrm{kg}$ were not included in the figure. 
$1147.1 \mathrm{mbsf})$. The coarseness and lack of bioturbation and evidence of soft-sediment deformation in one of the cores $(645 \mathrm{E}-$ 66R) suggest that the lowermost portion of Subunit IIIC was influenced by downslope processes. The landmass near Site 645 consists of granite and granitic gneiss. Rocks of this type often contain low concentrations of magnetic minerals. The influx of carbonate in the uppermost section of Subunit IIIC must reflect a source other than nearby land, and it is not of biogenic origin. Aksu and Piper (1987) suggested that the source of carbonate being deposited in Baffin Bay today may be derived from the Paleozoic and Mesozoic sedimentary rocks north of Site 645. This may also be the source of carbonate in the upper part of Subunit IIIC.

The development of geostrophic currents that are initially strong and then wane into Subunit IIIB has been postulated to explain the change in lithology from Subunit IIIC into Subunit IIIB (Arthur et al., this volume). As the geostrophic currents waned, magnetic minerals, because they are denser than matrix minerals (e.g., quartz and calcite/dolomite), would be deposited farther upcurrent; and the magnetic mineral concentration at the site of deposition would be reduced.

Through Subunits IIIB to the upper part of Subunit IIIA, the rock-magnetic and $\mathrm{CaCO}_{3}$ signatures appear as peaks superimposed on a low background. However, from $460 \mathrm{mbsf}$ to the top of Subunit IIIA, there is an abrupt increase in $X, X_{\mathrm{ARM}} / X$, HIRM, and carbonate content. Cremer and Legigan (this volume) also show an increase in the $>250 \mu \mathrm{m}$ bulk-sediment size fraction at this level.

Srivastava, Arthur, et al. (1987) suggested that the upper part of Subunit IIIA may contain ice-rafted material. The $X$ and carbonate signals of the upper part of Subunit IIIA, from 460 mbsf to the top of Subunit IIIA, resemble those of Unit II, which supports the hypothesis of early initiation of ice rafting. If this part of Subunit IIIA has been influenced by ice rafting, then from the age-depth profile of Site 645 (Srivastava, Arthur, et al., 1987) and the apparent location of the base of the Gauss magnetic normal at 460 mbsf (Clement et al., this volume), we infer an early-late Pliocene age (approximately $3.4 \mathrm{Ma}$ ) as the initiation of glaciation in the Northern Hemisphere.

The distinction between lithologic Units I and II is based on the decrease in detrital carbonate in Unit II. We do not see a distinct change in rock-magnetic parameters at the Unit I/Unit II boundary. However, there is an apparent change at the Subunit IA/Subunit IB boundary as $X$ and $X_{\mathrm{ARM}} / X$ show less fluctuation in Subunit IA. The change in carbonate content and rockmagnetic parameters probably reflects the increasing significance of ice rafting in Baffin Bay. The change in rock-magnetic signal at the Subunit IA/Subunit IB boundary probably reflects the increased coarseness of Subunit IA, coincidental with increased ice rafting.

\section{CONCLUSIONS}

From the data presented, we reach the following conclusions:

1. Whereas the concentration and grain size of magnetic minerals can be used to correlate lithologic changes in the upper part of Site 645 (APC cores), magnetic mineral variations cannot.

2. At Site 645 , variations in rock-magnetic parameters can be related to depositional changes that have occurred from the early-middle Miocene (Subunit IIIC to Subunit IA). The rock- magnetic parameters also reflect variations in carbonate content. Peaks at approximately $460 \mathrm{mbsf}$ in $X, X_{\mathrm{ARM}} / X$, HIRM, and carbonate content are interpreted to reflect an early episode of deposition by ice rafting at Site 645 that was initiated at approximately $3.4 \mathrm{Ma}$.

\section{ACKNOWLEDGMENTS}

This research was supported by USSAC PO\# 70760 Leg 105 . We thank J. Bloemendal and M. Arthur for reviewing this manuscript. We also thank Paula Weiss, A. Spies, and J. Corbin for assistance during sampling at the ODP Northeast Repository.

\section{REFERENCES}

Aksu, A., and Piper, D.J.W., 1987. Late Quaternary sedimentation in Baffin Bay. Can. J. Earth Sci., 24:1833-1846.

Bloemendal, J., Barton, C. E., and Radhakrishnamurthy, C., 1985. Correlation between Rayleigh loops and frequency-dependent and quadrature susceptibility: application to magnetic granulometry of rocks. J. Geophys. Res., 90:8789-8792.

Bloemendal, J., Lamb, B., and King, J. W., 1988. Paleoenvironmental implications of rock-magnetic properties of late Quaternary deepsea sediments from the eastern equatorial Atlantic. Paleoceanography, 3:61-88.

Collinson, D. W., 1983. Methods in Rock-Magnetism and Paleomagnetism: Bristol (Chapman and Hall), 1-445.

Doh, S.-J., King, and M. Leinen, 1988, A rock-magnetic study of giant piston Core LL44-GPC3 from the central North Pacific and its paleoceanographic implications. Paleoceanography, 3:89-112.

King, J. W., 1986. Paleomagnetic and rock-magnetic stratigraphy of Pigmy Basin, Deep Sea Drilling Project Site 619, Leg 96. In Coleman, A. H., and Meyer, A. W., et al., Init. Repts. DSDP, 96: Washington (U.S. Govt. Printing Office), 677-684.

King, J. W., Leskee, W., Marvin, J., and Banerjee, S. K., 1982a. Identification of magnetite and hematite in natural samples: a comparison of different rock magnetic methods. EOS, 63:917.

King, J., Banerjee, S. K., Marvin, J., and Özdemir, Ö., 1982b. A comparison of different magnetic methods for determining the relative grain size in natural materials: some results from lake sediments. Earth Planet. Sci. Lett., 59:404-419.

Martinson, D. G., Menke, W., and Stoffa, P., 1982. An inverse approach to signal correlation. J. Geophys. Res., 87:4807-4818.

Martinson, D. G., Pisias, N. G., Hays, J. D., Imbrie, J., Moore, T. C., and Shackleton, N. J., 1987. Age dating and the orbital theory of the Ice Ages: development of a high-resolution 0 to 300,000-year chronostratigraphy. Quat. Res., 27:1-29.

Molyneux, L., 1971. A complete result magnetometer for measuring the remanent magnetization of rocks. Geophys. J. Roy. Astr. Soc., 24: 429-433.

O'Reilly, W., 1984. Rock and Mineral Magnetism: Glasgow (Blacklie), $1-212$.

Robinson, S., 1986. The late Pleistocene paleoclimatic record of North Atlantic deep-sea sediments revealed by mineral-magnetic measurements. Phys. Earth Planet. Inter., 42:22-47.

Srivastava, S. P., Arthur, M., et al., 1987. Proc. ODP, Init. Repts., 105: College Station, TX (Ocean Drilling Program).

Stephenson, A., 1971. Single-domain grain distributions: I. a method for the determination of single-domain grain distributions. Phys. Earth Planet. Inter., 4:353-360.

Thompson, R., Bloemendal, J., Dearing, J. A., Oldfield, F., Rummery, T. J., Stober, J. C., and Turner, G. M., 1980. Environmental applications of magnetic measurements. Science, 207:481-486.

Thompson, R., and Oldfield, F., 1986. Environmental Magnetism: New York (Allen and Unwin), 1-227.

Date of initial receipt: 6 April 1988

Date of acceptance: 21 October 1988

Ms 105B-150 
APPENDIX

Rock-magnetic data collected for Site 645.

\begin{tabular}{|c|c|c|c|c|c|c|c|c|c|c|c|}
\hline Hole & Core : & & $\begin{array}{l}\text { Depth } \\
(\mathrm{cm})\end{array}$ & $\begin{array}{c}\text { SBD } \\
\text { (mbsf) }\end{array}$ & $\begin{array}{c}\text { MASS } \\
(\mathrm{g})\end{array}$ & $\underset{10-6 \mathrm{~m}^{3} / \mathrm{kg}}{\mathrm{X}}$ & $\underset{10-6 \mathrm{~m}^{3} / \mathrm{kg}}{X_{\mathrm{ARM}}}$ & $\mathrm{X}_{\text {ARM }} / \mathrm{X}$ & $\begin{array}{c}\text { SIRM } \\
(\mathrm{mA} / \mathrm{m} \mathrm{kg})\end{array}$ & $\underset{(\mathrm{mA} / \mathrm{m} \mathrm{kg})}{\mathrm{HIRM}}$ & $\mathrm{S}$ \\
\hline $645 A$ & $1 \mathrm{H}$ & 1 & 6 & 0.06 & 7.44 & 35.52 & 3171.10 & 89.28 & 9.52 & 3.30 & 0.995 \\
\hline $645 A$ & $1 \mathrm{H}$ & 1 & 16 & 0.16 & 8.53 & 53.60 & 2158.06 & 40.26 & 12.87 & 16.55 & 0.978 \\
\hline $645 A$ & $1 \mathrm{H}$ & 1 & 26 & 0.26 & 9.58 & 49.32 & 1807.18 & 36.64 & 13.09 & 8.16 & 0.988 \\
\hline $645 A$ & IH & 1 & 36 & 0.36 & 9.94 & 50.44 & 1050.90 & 20.84 & 11.02 & 8.07 & 0.985 \\
\hline $645 A$ & $1 \mathrm{H}$ & 1 & 46 & 0.46 & 10.96 & 49.46 & 535.30 & 10.82 & 9.84 & 9.29 & 0.979 \\
\hline $645 A$ & $1 \mathrm{H}$ & 1 & 56 & 0.56 & 8.67 & 56.32 & 1910.94 & 33.93 & 10.76 & 9.34 & 0.985 \\
\hline $645 A$ & $1 \mathrm{H}$ & 1 & 66 & 0.66 & 9.86 & 77.53 & 3736.86 & 48.20 & 17.26 & 16.52 & 0.981 \\
\hline $645 \mathrm{~A}$ & $1 \mathrm{H}$ & 1 & 73 & 0.73 & 9.60 & 95.84 & 3365.26 & 35.11 & 15.13 & 19.72 & 0.975 \\
\hline $645 A$ & $1 \mathrm{H}$ & 1 & 83 & 0.83 & 9.46 & 55.86 & 1823.07 & 32.63 & 7.74 & 14.26 & 0.965 \\
\hline $645 A$ & $1 \mathrm{H}$ & 1 & 94 & 0.94 & 10.52 & 80.46 & 1825.55 & 22.69 & 10.55 & 16.47 & 0.967 \\
\hline $645 A$ & $1 \mathrm{H}$ & 1 & 104 & 1.04 & 8.02 & 84.45 & 7623.22 & 90.27 & 27.17 & 20.23 & 0.988 \\
\hline $645 A$ & $1 \mathrm{H}$ & 1 & 114 & 1.14 & 7.99 & 65.93 & 9356.07 & 141.91 & 28.18 & 11.74 & 0.993 \\
\hline $645 A$ & $1 \mathrm{H}$ & 1 & 121 & 1.21 & 7.80 & 42.95 & 5620.09 & 130.87 & 20.22 & 13.33 & 0.990 \\
\hline $645 A$ & $1 \mathrm{H}$ & 1 & 127 & 1.27 & 7.75 & 33.44 & 5377.69 & 160.83 & 16.61 & 19.45 & 0.982 \\
\hline $645 A$ & $1 \mathrm{H}$ & 1 & 142 & 1.42 & 8.74 & 54.84 & 5316.49 & 96.95 & 24.08 & 2.49 & 0.998 \\
\hline $645 A$ & $1 \mathrm{H}$ & 2 & 7 & 1.57 & 9.76 & 73.16 & 4441.65 & 60.71 & 24.79 & 16.99 & 0.987 \\
\hline $645 A$ & $1 \mathrm{H}$ & 2 & 19 & 1.69 & 8.97 & 92.60 & 6087.99 & 65.74 & 31.25 & 22.80 & 0.987 \\
\hline $645 A$ & $1 \mathrm{H}$ & 2 & 26 & 1.76 & 9.50 & 107.98 & 5810.37 & 53.81 & 34.41 & 1.67 & 0.999 \\
\hline $645 \mathrm{~A}$ & $1 \mathrm{H}$ & 2 & 35 & 1.85 & 9.56 & 113.99 & 5323.82 & 46.70 & 34.60 & 0.33 & 1.000 \\
\hline $645 A$ & $1 \mathrm{H}$ & 2 & 57 & 2.07 & 10.28 & 109.75 & 4968.54 & 45.27 & 25.09 & 6.88 & 0.994 \\
\hline $645 A$ & $1 \mathrm{H}$ & 2 & 67 & 2.17 & 9.65 & 117.68 & 5280.42 & 44.87 & 26.08 & 10.10 & 0.993 \\
\hline $645 A$ & $1 \mathrm{H}$ & 2 & 77 & 2.27 & 10.01 & 106.50 & 4728.06 & 44.40 & 22.54 & 11.70 & 0.990 \\
\hline $645 A$ & $1 \mathrm{H}$ & 2 & 87 & 2.37 & 9.66 & 100.28 & 4490.75 & 44.78 & 20.50 & 17.11 & 0.984 \\
\hline $645 A$ & $1 \mathrm{H}$ & 2 & 97 & 2.47 & 10.18 & 114.83 & 4837.05 & 42.12 & 22.66 & 2.00 & 0.998 \\
\hline $645 A$ & $1 \mathrm{H}$ & 2 & 107 & 2.57 & 9.87 & 110.36 & 4784.05 & 43.35 & 21.48 & 13.39 & 0.988 \\
\hline $645 A$ & $1 \mathrm{H}$ & 2 & 115 & 2.65 & 10.33 & 107.69 & 4086.99 & 37.95 & 19.69 & 24.39 & 0.974 \\
\hline $645 A$ & $1 \mathrm{H}$ & 2 & 124 & 2.74 & 10.44 & 105.63 & 4402.88 & 41.68 & 21.15 & 28.12 & 0.972 \\
\hline $645 A$ & $1 \mathrm{H}$ & 2 & 134 & 2.84 & 10.26 & 159.77 & 4550.65 & 28.48 & 22.65 & 16.01 & 0.985 \\
\hline $645 A$ & $1 \mathrm{H}$ & 2 & 144 & 2.94 & 10.07 & 117.44 & 4644.25 & 39.55 & 21.39 & 5.27 & 0.995 \\
\hline $645 A$ & $1 \mathrm{H}$ & 3 & 6 & 3.06 & 10.07 & 116.87 & 4828.26 & 41.31 & 22.24 & 20.04 & 0.982 \\
\hline $645 A$ & $1 \mathrm{H}$ & 3 & 16 & 3.16 & 10.31 & 110.70 & 4535.16 & 40.97 & 21.52 & 10.61 & 0.990 \\
\hline $645 A$ & $1 \mathrm{H}$ & 3 & 26 & 3.26 & 10.41 & 105.77 & 4658.98 & 44.05 & 21.36 & 7.87 & 0.992 \\
\hline $645 A$ & $1 \mathrm{H}$ & 3 & 36 & 3.36 & 10.58 & 111.78 & 4727.68 & 42.30 & 21.93 & 2.95 & 0.997 \\
\hline $645 A$ & 1H & 3 & 46 & 3.46 & 9.63 & 88.19 & 3973.01 & 45.05 & 19.04 & 1.16 & 0.999 \\
\hline $645 A$ & $1 \mathrm{H}$ & 3 & 56 & 3.56 & 10.05 & 92.22 & 4104.35 & 44.50 & 18.43 & 19.19 & 0.979 \\
\hline $645 A$ & $1 \mathrm{H}$ & 3 & 66 & 3.66 & 10.01 & 124.64 & 5005.17 & 40.16 & 22.98 & 9.35 & 0.992 \\
\hline $645 A$ & $1 \mathrm{H}$ & 3 & 76 & 3.76 & 10.22 & 113.17 & 5032.62 & 44.47 & 22.60 & 30.37 & 0.973 \\
\hline $645 \mathrm{~A}$ & $1 \mathrm{H}$ & 3 & 86 & 3.86 & 10.47 & 88.85 & 3580.70 & 40.30 & 16.85 & 25.02 & 0.969 \\
\hline $645 \mathrm{~A}$ & $1 \mathrm{H}$ & 3 & 93 & 3.93 & 10.57 & 92.56 & 3632.52 & 39.25 & 17.85 & 28.67 & 0.966 \\
\hline $645 A$ & $1 \mathrm{H}$ & 3 & 104 & 4.04 & 9.18 & 101.45 & 4739.52 & 46.72 & 19.73 & 13.10 & 0.988 \\
\hline $645 A$ & $1 \mathrm{H}$ & 3 & 114 & 4.14 & 10.11 & 93.86 & 3585.56 & 38.20 & 17.26 & 15.02 & 0.982 \\
\hline $645 A$ & $1 \mathrm{H}$ & 3 & 124 & 4.24 & 10.14 & 99.83 & 3824.35 & 38.31 & 18.50 & 41.00 & 0.955 \\
\hline $645 A$ & $1 \mathrm{H}$ & 3 & 134 & 4.34 & 10.32 & 100.61 & 3907.50 & 38.84 & 19.09 & 26.69 & 0.971 \\
\hline $645 A$ & $1 \mathrm{H}$ & 3 & 144 & 4.44 & 10.58 & 71.56 & 3182.86 & 44.48 & 14.47 & 33.24 & 0.951 \\
\hline $645 A$ & $1 \mathrm{H}$ & 4 & 2 & 4.52 & 10.75 & 73.64 & 2813.02 & 38.20 & 13.78 & 22.47 & 0.965 \\
\hline $645 A$ & $1 \mathrm{H}$ & 4 & 12 & 4.62 & 11.00 & 78.29 & 3063.27 & 39.13 & 16.28 & 22.85 & 0.969 \\
\hline $645 A$ & $1 \mathrm{H}$ & 4 & 22 & 4.72 & 10.80 & 73.14 & 2805.70 & 38.36 & 14.59 & 22.75 & 0.966 \\
\hline $645 A$ & $1 \mathrm{H}$ & 4 & 28 & 4.78 & 10.92 & 62.70 & 2272.53 & 35.29 & 12.33 & 20.23 & 0.964 \\
\hline $645 C$ & $1 \mathrm{H}$ & 1 & 12 & 0.12 & 6.24 & 20.94 & 2507.50 & 119.76 & 6.48 & 10.82 & 0.979 \\
\hline $645 C$ & $1 \mathrm{H}$ & 1 & 20 & 0.20 & 7.20 & 29.14 & 2534.51 & 86.98 & 9.66 & 43.06 & 0.936 \\
\hline $645 C$ & $1 \mathrm{H}$ & 1 & 30 & 0.30 & 5.53 & 47.25 & 1946.88 & 41.20 & 10.37 & 48.15 & 0.949 \\
\hline $645 C$ & $1 \mathrm{H}$ & 1 & 40 & 0.40 & 8.63 & 37.70 & 678.36 & 17.99 & 8.71 & 20.86 & 0.959 \\
\hline $645 \mathrm{C}$ & $1 \mathrm{H}$ & 1 & 50 & 0.50 & 9.66 & 69.97 & 1009.98 & 14.44 & 12.18 & 18.63 & 0.970 \\
\hline $645 \mathrm{C}$ & $1 \mathrm{H}$ & 1 & 60 & 0.60 & 10.37 & 46.64 & 346.12 & 7.42 & 8.30 & 33.03 & 0.917 \\
\hline $645 \mathrm{C}$ & $1 \mathrm{H}$ & 1 & 70 & 0.70 & 8.66 & 73.40 & 1881.60 & 25.63 & 11.00 & 12.70 & 0.980 \\
\hline $645 \mathrm{C}$ & $1 \mathrm{H}$ & 1 & 80 & 0.80 & 7.59 & 79.12 & 4169.87 & 52.70 & 16.54 & 18.77 & 0.983 \\
\hline $645 C$ & 1H & 1 & 90 & 0.90 & 10.19 & 62.01 & 1359.27 & 21.92 & 10.18 & 31.89 & 0.936 \\
\hline
\end{tabular}


Appendix (continued)

\begin{tabular}{|c|c|c|c|c|c|c|c|c|c|c|c|}
\hline Hole & Core S & & $\begin{array}{l}\text { Depth } \\
\text { (cm) }\end{array}$ & $\begin{array}{c}\text { SBD } \\
\text { (mbsf) }\end{array}$ & $\underset{(\mathrm{g})}{\text { MASS }}$ & $\underset{10^{-6} \mathrm{~m}^{3} / \mathrm{kg}}{\mathrm{X}}$ & $\underset{10-6 \mathrm{~m}^{3} / \mathrm{kg}}{\mathrm{X}_{\mathrm{ARM}}}$ & $X_{\text {ARM }} / X$ & $\underset{(\mathrm{mA} / \mathrm{m} \mathrm{kg})}{\operatorname{SIRM}}$ & $\underset{(\mathrm{mA} / \mathrm{m} \mathrm{kg})}{\mathrm{HIRM}}$ & S \\
\hline $645 \mathrm{C}$ & $1 \mathrm{H} 1$ & 11 & 100 & 1.00 & 8.44 & 75.02 & 4164.20 & 55.51 & 21.40 & 40.72 & 0.968 \\
\hline $645 \mathrm{C}$ & $1 \mathrm{H} 1$ & 11 & 110 & 1.10 & 7.96 & 67.39 & 6075.23 & 90.15 & 22.24 & 60.46 & 0.957 \\
\hline $645 \mathrm{C}$ & $1 \mathrm{H} 1$ & 11 & 120 & 1.20 & 8.53 & 79.97 & 4256.33 & 53.22 & 21.91 & 4.40 & 0.997 \\
\hline $645 \mathrm{C}$ & $1 \mathrm{H} 1$ & 11 & 130 & 1.30 & 7.74 & 40.25 & 1541.97 & 38.31 & 19.92 & 26.65 & 0.979 \\
\hline $645 C$ & $1 \mathrm{H} 2$ & 2 & 10 & 1.60 & 8.43 & 37.11 & 790.70 & 21.31 & 11.96 & 51.16 & 0.928 \\
\hline $645 \mathrm{C}$ & 1H 2 & 2 & 20 & 1.70 & 7.53 & 61.40 & 2724.54 & 44.38 & 19.76 & 86.99 & 0.934 \\
\hline $645 C$ & 1H 2 & 2 & 31 & 1.81 & 8.61 & 103.89 & 3290.80 & 31.68 & 29.45 & 15.97 & 0.991 \\
\hline $645 \mathrm{C}$ & $1 \mathrm{H} 2$ & 2 & 40 & 1.90 & 9.43 & 137.88 & 3370.51 & 24.44 & 36.64 & 23.86 & 0.988 \\
\hline $645 \mathrm{C}$ & $1 \mathrm{H} 2$ & 2 & 60 & 2.10 & 10.39 & 129.50 & 2758.17 & 21.30 & 41.10 & 36.09 & 0.982 \\
\hline $645 \mathrm{C}$ & 1H 2 & 2 & 80 & 2.30 & 10.09 & 131.36 & 2532.75 & 19.28 & 22.72 & 9.91 & 0.991 \\
\hline $645 \mathrm{C}$ & 1H 2 & 2 & 90 & 2.40 & 9.58 & 113.17 & 2264.34 & 20.01 & 20.95 & 19.57 & 0.982 \\
\hline $645 \mathrm{C}$ & 1H 2 & 21 & 100 & 2.50 & 9.99 & 91.30 & 1827.25 & 20.01 & 16.21 & 17.52 & 0.978 \\
\hline $645 \mathrm{C}$ & $1 \mathrm{H} 2$ & 21 & 120 & 2.70 & 10.50 & 56.59 & 1316.10 & 23.26 & 12.38 & 35.71 & 0.939 \\
\hline $645 C$ & $1 \mathrm{H} 2$ & 21 & 130 & 2.80 & 9.00 & 62.81 & 1310.72 & 20.87 & 10.88 & 10.42 & 0.983 \\
\hline $645 \mathrm{C}$ & $1 \mathrm{H} 3$ & 3 & 2 & 3.02 & 11.10 & 68.13 & 1382.30 & 20.29 & 13.22 & 32.66 & 0.945 \\
\hline $645 \mathrm{C}$ & $1 \mathrm{H}$ & 3 & 10 & 3.10 & 10.69 & 70.63 & 1804.43 & 25.55 & 13.34 & 29.23 & 0.953 \\
\hline $645 \mathrm{C}$ & $1 \mathrm{H} 3$ & 3 & 20 & 3.20 & 9.58 & 47.86 & 1527.98 & 31.92 & 8.76 & 30.66 & 0.933 \\
\hline $645 \mathrm{C}$ & $1 \mathrm{H}$ & 3 & 30 & 3.30 & 9.24 & 109.72 & 3317.45 & 30.24 & 21.31 & 117.01 & 0.899 \\
\hline $645 \mathrm{C}$ & $1 \mathrm{H}$ & 3 & 40 & 3.40 & 7.87 & 115.57 & 3463.95 & 29.97 & 19.35 & 54.00 & 0.956 \\
\hline $645 C$ & 1H & 3 & 50 & 3.50 & 9.65 & 119.12 & 3215.56 & 26.99 & 22.06 & 47.93 & 0.958 \\
\hline $645 \mathrm{C}$ & 1H & 3 & 80 & 3.80 & 9.69 & 71.05 & 1990.56 & 28.02 & 11.65 & 23.22 & 0.961 \\
\hline $645 \mathrm{C}$ & $1 \mathrm{H}$ & 3 & 90 & 3.90 & 9.16 & 101.76 & 2851.92 & 28.02 & 18.30 & 64.14 & 0.936 \\
\hline $645 \mathrm{C}$ & $1 \mathrm{H}$ & 31 & 100 & 4.00 & 8.86 & 128.18 & 5161.40 & 40.27 & 24.67 & 9.88 & 0.993 \\
\hline $645 \mathrm{C}$ & $1 \mathrm{H}$ & 3 & 130 & 4.30 & 9.76 & 103.49 & 1621.81 & 15.67 & 12.38 & 28.18 & 0.956 \\
\hline $645 \mathrm{C}$ & $3 \mathrm{H}$ & 1 & 20 & 14.20 & 9.47 & 78.53 & 2156.68 & 27.46 & 12.31 & 9.24 & 0.986 \\
\hline $645 \mathrm{C}$ & 3H : & 1 & 30 & 14.30 & 10.38 & 74.31 & 3069.61 & 41.31 & 14.90 & 25.89 & 0.964 \\
\hline $645 \mathrm{C}$ & $3 \mathrm{H}$ & 1 & 50 & 14.50 & 12.59 & 64.46 & 2265.10 & 35.14 & 24.16 & 20.84 & 0.978 \\
\hline $645 \mathrm{C}$ & $3 \mathrm{H}$ & 1 & 60 & 14.60 & 9.53 & 126.29 & 4468.83 & 35.39 & 26.16 & 38.69 & 0.972 \\
\hline $645 \mathrm{C}$ & $3 \mathrm{H}$ & 1 & 70 & 14.70 & 11.83 & 35.36 & 1038.09 & 29.36 & 6.59 & 8.77 & 0.969 \\
\hline $645 \mathrm{C}$ & $3 \mathrm{H}$ & 1 & 83 & 14.83 & 10.86 & 37.60 & 914.55 & 24.33 & 5.59 & 12.78 & 0.950 \\
\hline $645 C$ & $3 \mathrm{H}$ & 1 & 90 & 14.90 & 11.09 & 34.32 & 973.23 & 28.35 & 6.11 & 15.33 & 0.944 \\
\hline $645 \mathrm{C}$ & $3 \mathrm{H}$ & $1:$ & 100 & 15.00 & 10.19 & 30.70 & 884.76 & 28.82 & 4.88 & 13.98 & 0.942 \\
\hline $645 \mathrm{C}$ & $3 \mathrm{H}$ & 1. & 110 & 15.10 & 9.42 & 95.89 & 4467.31 & 46.59 & 18.60 & 37.02 & 0.962 \\
\hline $645 \mathrm{C}$ & $3 \mathrm{H}$ & $1:$ & 120 & 15.20 & 9.61 & 91.12 & 4627.72 & 50.79 & 22.85 & 29.92 & 0.975 \\
\hline $645 \mathrm{C}$ & $3 \mathrm{H}$ & $1:$ & 130 & 15.30 & 9.27 & 125.90 & 5403.40 & 42.92 & 21.00 & 34.39 & 0.970 \\
\hline $645 \mathrm{C}$ & $3 \mathrm{H}$ & 1 & 140 & 15.40 & 11.08 & 62.47 & 1443.37 & 23.10 & 8.69 & 7.90 & 0.980 \\
\hline $645 \mathrm{C}$ & $3 \mathrm{H}$ & 2 & 0 & 15.50 & 10.32 & 60.50 & 1947.84 & 32.20 & 9.66 & 17.56 & 0.962 \\
\hline $645 \mathrm{C}$ & $3 \mathrm{H}$ & 2 & 10 & 15.60 & 10.86 & 70.10 & 2207.26 & 31.49 & 10.35 & 18.99 & 0.960 \\
\hline $645 \mathrm{C}$ & $3 \mathrm{H}$ & 2 & 20 & 15.70 & 10.91 & 87.05 & 3719.33 & 42.72 & 21.75 & 15.47 & 0.984 \\
\hline $645 \mathrm{C}$ & $3 \mathrm{H}$ & 2 & 50 & 16.00 & 10.05 & 79.75 & 4649.24 & 58.30 & 19.10 & 20.52 & 0.978 \\
\hline $645 \mathrm{C}$ & $3 \mathrm{H}$ & 2 & 60 & 16.10 & 9.78 & 68.34 & 2059.70 & 30.14 & 14.50 & 70.94 & 0.904 \\
\hline $645 \mathrm{C}$ & $3 \mathrm{H}$ & 2 & 70 & 16.20 & 9.99 & 59.98 & 1888.00 & 31.47 & 10.80 & 17.52 & 0.968 \\
\hline $645 \mathrm{C}$ & $3 \mathrm{H}$ & 2 & 80 & 16.30 & 11.06 & 47.82 & 1255.75 & 26.26 & 8.74 & 2.83 & 0.993 \\
\hline $645 \mathrm{C}$ & $3 \mathrm{H} 2$ & 2 & 90 & 16.40 & 10.49 & 60.60 & 1262.29 & 20.83 & 8.40 & 13.11 & 0.967 \\
\hline $645 \mathrm{C}$ & $3 \mathrm{H}$ & 23 & 102 & 16.52 & 10.18 & 45.91 & 1011.34 & 22.03 & 6.92 & 13.26 & 0.961 \\
\hline $645 \mathrm{C}$ & $3 \mathrm{H}$ & 21 & 120 & 16.70 & 10.39 & 37.48 & 1264.18 & 33.73 & 8.11 & 15.40 & 0.961 \\
\hline $645 \mathrm{C}$ & $3 \mathrm{H}$ & 3 & 44 & 17.44 & 9.20 & 96.41 & 2990.50 & 31.02 & 17.44 & 35.33 & 0.963 \\
\hline $645 \mathrm{C}$ & 3H & 3 & 54 & 17.54 & 8.96 & 178.91 & 3648.16 & 20.39 & 20.82 & 46.74 & 0.960 \\
\hline $645 \mathrm{C}$ & $3 \mathrm{H}$ & 3 & 64 & 17.64 & 9.26 & 96.73 & 5056.56 & 52.27 & 25.39 & 12.83 & 0.991 \\
\hline $645 \mathrm{C}$ & $3 \mathrm{H}$ & 3 & 71 & 17.71 & 10.67 & 46.74 & 906.26 & 19.39 & 8.25 & 16.40 & 0.958 \\
\hline $645 \mathrm{C}$ & $3 \mathrm{H}$ & 3 & 94 & 17.94 & 10.83 & 38.74 & 795.66 & 20.54 & 6.47 & 17.77 & 0.940 \\
\hline $645 \mathrm{C}$ & $3 \mathrm{H}$ & 3 & 101 & 18.01 & 10.69 & 52.65 & 1023.70 & 19.44 & 8.33 & 12.86 & 0.967 \\
\hline $645 \mathrm{C}$ & 3H & 3 & 105 & 18.05 & 7.74 & 52.43 & 1857.10 & 35.42 & 8.54 & 14.53 & 0.974 \\
\hline $645 \mathrm{C}$ & $3 \mathrm{H}$ & $3:$ & 124 & 18.24 & 9.55 & 135.63 & 2963.90 & 21.85 & 20.95 & 15.71 & 0.986 \\
\hline $645 C$ & 3H & $3:$ & 141 & 18.41 & 9.01 & 121.17 & 2249.94 & 18.57 & 14.96 & 2.77 & 0.997 \\
\hline $645 \mathrm{C}$ & $3 \mathrm{H}$ & 4 & 25 & 18.75 & 10.30 & 145.39 & 2473.40 & 17.01 & 18.81 & 16.99 & 0.981 \\
\hline $645 \mathrm{C}$ & 3H & 5 & 0 & 20.00 & 9.93 & 114.12 & 4780.71 & 41.89 & 26.59 & 17.62 & 0.987 \\
\hline
\end{tabular}


Appendix (continued)

\begin{tabular}{|c|c|c|c|c|c|c|c|c|c|c|c|}
\hline Hole & Core S & & $\begin{array}{l}\text { Depth } \\
(\mathrm{cm})\end{array}$ & $\begin{array}{c}\text { SBD } \\
\text { (mbsf) }\end{array}$ & $\begin{array}{c}\text { MASS } \\
(\mathrm{g})\end{array}$ & $\underset{10-6 \mathrm{~m}^{3} / \mathrm{kg}}{\mathrm{X}}$ & $\underset{10-6 \mathrm{~m}^{3} / \mathrm{kg}}{\mathrm{X}_{\mathrm{ARM}}}$ & $\mathrm{X}_{\text {ARM }} / \mathrm{X}$ & $\begin{array}{c}\text { SIRM } \\
(\mathrm{mA} / \mathrm{m} \mathrm{kg})\end{array}$ & $\begin{array}{c}\text { HIRM } \\
(\mathrm{mA} / \mathrm{m} \mathrm{kg})\end{array}$ & $\mathrm{s}$ \\
\hline $645 \mathrm{C}$ & $3 \mathrm{H}$ & 5 & 16 & 20.16 & 10.54 & 38.98 & 1516.60 & 38.91 & 10.21 & 10.08 & 0.979 \\
\hline $645 \mathrm{C}$ & $3 \mathrm{H}$ & 5 & 24 & 20.24 & 11.41 & 41.18 & 781.09 & 18.97 & 8.20 & 9.86 & 0.973 \\
\hline $645 C$ & 3H & 5 & 35 & 20.35 & 10.50 & 54.68 & 909.25 & 16.63 & 9.00 & 15.48 & 0.964 \\
\hline $645 C$ & 3H & 5 & 44 & 20.44 & 11.55 & 71.46 & 1092.95 & 15.29 & 10.66 & 12.99 & 0.972 \\
\hline $645 C$ & $3 \mathrm{H}$ & 5 & 73 & 20.73 & 10.92 & 77.08 & 1277.89 & 16.58 & 10.86 & 26.33 & 0.947 \\
\hline $645 C$ & $3 \mathrm{H}$ & 5 & 82 & 20.82 & 8.74 & 69.14 & 2354.54 & 34.06 & 13.79 & 14.30 & 0.982 \\
\hline $645 C$ & $3 \mathrm{H}$ & 5 & 97 & 20.97 & 9.65 & 65.35 & 1823.25 & 27.90 & 11.32 & 12.31 & 0.979 \\
\hline $645 F$ & $1 \mathrm{H}$ & 1 & 2 & 0.02 & 7.42 & 25.99 & 3531.64 & 135.87 & 8.93 & 21.67 & 0.964 \\
\hline $645 F$ & $1 \mathrm{H}$ & 1 & 12 & 0.12 & 7.43 & 53.37 & 2437.49 & 45.67 & 13.47 & 20.91 & 0.977 \\
\hline $645 F$ & $1 \mathrm{H}$ & 1 & 22 & 0.22 & 9.92 & 59.30 & 1742.96 & 29.39 & 14.04 & 17.75 & 0.975 \\
\hline $645 F$ & $1 \mathrm{H}$ & 1 & 32 & 0.32 & 10.62 & 60.86 & 911.45 & 14.98 & 11.37 & 12.29 & 0.977 \\
\hline $645 F$ & $1 \mathrm{H}$ & 1 & 38 & 0.38 & 10.96 & 53.21 & 705.28 & 13.26 & 11.15 & 14.99 & 0.971 \\
\hline $645 F$ & $1 \mathrm{H}$ & 1 & 48 & 0.48 & 9.73 & 104.78 & 3936.69 & 37.57 & 24.50 & 29.19 & 0.977 \\
\hline $645 F$ & $1 \mathrm{H}$ & 1 & 58 & 0.58 & 8.91 & 76.62 & 2350.98 & 30.68 & 10.23 & 16.99 & 0.970 \\
\hline $645 F$ & $1 \mathrm{H}$ & 1 & 68 & 0.68 & 11.16 & 59.24 & 1731.44 & 29.23 & 10.05 & 21.49 & 0.952 \\
\hline $645 F$ & $1 \mathrm{H}$ & 1 & 74 & 0.74 & 9.16 & 74.84 & 4072.77 & 54.42 & 18.98 & 25.86 & 0.975 \\
\hline $645 F$ & $1 \mathrm{H}$ & 1 & 83 & 0.83 & 8.29 & 67.74 & 4736.44 & 69.92 & 14.71 & 19.18 & 0.978 \\
\hline $645 F$ & $1 \mathrm{H}$ & 1 & 93 & 0.93 & 7.62 & 42.41 & 6339.28 & 149.28 & 23.16 & 27.02 & 0.982 \\
\hline $645 F$ & $1 \mathrm{H}$ & 2 & 12 & 1.62 & 9.81 & 65.94 & 2242.23 & 34.00 & 16.09 & 15.81 & 0.981 \\
\hline $645 F$ & $1 \mathrm{H}$ & 2 & 22 & 1.72 & 8.90 & 58.04 & 5067.61 & 87.31 & 26.85 & 24.19 & 0.984 \\
\hline $645 F$ & $1 \mathrm{H}$ & 2 & 32 & 1.82 & 8.66 & 90.20 & 6111.39 & 67.75 & 33.01 & 0.20 & 1.000 \\
\hline $645 F$ & $1 \mathrm{H}$ & 2 & 52 & 2.02 & 8.38 & 115.35 & 7148.21 & 61.97 & 37.79 & 16.98 & 0.992 \\
\hline $645 F$ & $1 \mathrm{H}$ & 2 & 104 & 2.54 & 9.75 & 148.04 & 5848.22 & 39.50 & 24.46 & 15.30 & 0.988 \\
\hline $645 F$ & $1 \mathrm{H}$ & 2 & 114 & 2.64 & 10.09 & 160.98 & 5381.59 & 33.43 & 27.93 & 13.03 & 0.991 \\
\hline $645 F$ & $1 \mathrm{H}$ & 2 & 124 & 2.74 & 9.66 & 157.67 & 5477.18 & 34.74 & 26.22 & 7.66 & 0.994 \\
\hline $645 F$ & $1 \mathrm{H}$ & 2 & 134 & 2.84 & 9.28 & 165.29 & 6700.80 & 40.54 & 25.53 & 6.93 & 0.995 \\
\hline $645 F$ & $1 \mathrm{H}$ & 3 & 3 & 3.03 & 9.77 & 192.05 & 6152.56 & 32.04 & 32.08 & 3.25 & 0.998 \\
\hline $645 F$ & $1 \mathrm{H}$ & 3 & 13 & 3.13 & 9.92 & 184.68 & 6546.47 & 35.45 & 31.28 & 11.17 & 0.993 \\
\hline $645 F$ & $1 \mathrm{H}$ & 3 & 23 & 3.23 & 10.15 & 189.01 & 5266.11 & 27.86 & 36.44 & 111.65 & 0.938 \\
\hline $645 F$ & IH & 3 & 33 & 3.33 & 9.45 & 157.14 & 4073.68 & 25.92 & 25.46 & 16.34 & 0.988 \\
\hline $645 F$ & $1 \mathrm{H}$ & 3 & 43 & 3.43 & 9.62 & 89.13 & 2888.27 & 32.41 & 16.23 & 14.40 & 0.983 \\
\hline $645 F$ & $1 \mathrm{H}$ & 3 & 53 & 3.53 & 9.24 & 75.92 & 3155.52 & 41.56 & 12.29 & 13.71 & 0.979 \\
\hline $645 F$ & $1 \mathrm{H}$ & 3 & 64 & 3.64 & 10.98 & 72.39 & 3265.99 & 45.12 & 13.25 & 19.75 & 0.967 \\
\hline $645 F$ & $1 \mathrm{H}$ & 3 & 74 & 3.74 & 11.19 & 82.11 & 3083.00 & 37.55 & 14.96 & 9.32 & 0.986 \\
\hline $645 F$ & $1 \mathrm{H}$ & 3 & 82 & 3.82 & 11.04 & 80.19 & 0.00 & 0.00 & 14.40 & 8.38 & 0.987 \\
\hline $645 G$ & $1 \mathrm{H}$ & 2 & 11 & 1.61 & 9.19 & 129.27 & 4853.29 & 37.54 & 19.43 & 20.12 & 0.981 \\
\hline $645 G$ & $1 \mathrm{H}$ & 2 & 25 & 1.75 & 9.32 & 117.59 & 4937.24 & 41.99 & 20.15 & 29.38 & 0.973 \\
\hline $645 G$ & $1 \mathrm{H}$ & 2 & 34 & 1.84 & 9.85 & 129.60 & 5258.53 & 40.57 & 22.73 & 24.74 & 0.979 \\
\hline $645 G$ & $1 \mathrm{H}$ & 2 & 45 & 1.95 & 9.97 & 123.54 & 5460.22 & 44.20 & 23.48 & 28.00 & 0.976 \\
\hline $645 G$ & $1 \mathrm{H}$ & 2 & 52 & 2.02 & 9.74 & 125.93 & 4935.35 & 39.19 & 21.74 & 28.21 & 0.975 \\
\hline $645 G$ & $1 \mathrm{H}$ & 2 & 64 & 2.14 & 10.16 & 84.23 & 3119.81 & 37.04 & 15.36 & 20.68 & 0.973 \\
\hline $645 G$ & IH & 2 & 74 & 2.24 & 10.69 & 91.42 & 3858.98 & 42.21 & 17.71 & 26.10 & 0.968 \\
\hline $645 G$ & $1 \mathrm{H}$ & 2 & 85 & 2.35 & 10.48 & 89.19 & 3618.28 & 40.57 & 17.17 & 27.94 & 0.966 \\
\hline $645 G$ & $1 \mathrm{H}$ & 2 & 93 & 2.43 & 10.33 & 79.49 & 3415.92 & 42.97 & 14.85 & 21.68 & 0.970 \\
\hline $645 G$ & $1 \mathrm{H}$ & 2 & 108 & 2.58 & 10.13 & 96.59 & 3628.58 & 37.57 & 22.03 & 24.17 & 0.978 \\
\hline $645 G$ & $1 \mathrm{H}$ & 2 & 113 & 2.63 & 8.44 & 222.91 & 7102.75 & 31.86 & 29.28 & 19.77 & 0.989 \\
\hline $645 G$ & $1 \mathrm{H}$ & 2 & 130 & 2.80 & 10.18 & 107.55 & 5345.00 & 49.70 & 21.27 & 22.26 & 0.979 \\
\hline $645 G$ & $1 \mathrm{H}$ & 3 & 9 & 3.09 & 7.98 & 115.42 & 8369.23 & 72.51 & 26.01 & 50.53 & 0.969 \\
\hline $645 G$ & $1 \mathrm{H}$ & 3 & 33 & 3.33 & 10.19 & 55.11 & 2184.01 & 39.63 & 11.75 & 21.49 & 0.963 \\
\hline $645 G$ & $1 \mathrm{H}$ & 3 & 44 & 3.44 & 9.66 & 62.90 & 2437.65 & 38.75 & 11.50 & 18.22 & 0.969 \\
\hline $645 G$ & $1 \mathrm{H}$ & 3 & 52 & 3.52 & 9.73 & 74.12 & 2032.13 & 27.42 & 12.45 & 22.72 & 0.964 \\
\hline $645 G$ & $1 \mathrm{H}$ & 3 & 67 & 3.67 & 10.03 & 57.98 & 1891.31 & 32.62 & 11.12 & 19.98 & 0.964 \\
\hline $645 G$ & 1н & 3 & 74 & 3.74 & 10.72 & 66.06 & 2305.14 & 34.90 & 12.64 & 21.64 & 0.963 \\
\hline $645 G$ & $1 \mathrm{H}$ & 3 & 84 & 3.84 & 10.06 & 59.38 & 2708.37 & 45.61 & 12.46 & 22.85 & 0.963 \\
\hline $645 G$ & $1 \mathrm{H}$ & 3 & 93 & 3.93 & 8.44 & 128.98 & 9221.31 & 71.49 & 30.31 & 34.40 & 0.981 \\
\hline $645 G$ & 1H & 3 & 113 & 4.13 & 8.40 & 82.54 & 6650.07 & 80.56 & 23.36 & 27.86 & 0.980 \\
\hline $645 G$ & $1 \mathrm{H}$ & 3 & 129 & 4.29 & 9.86 & 71.82 & 4077.05 & 56.77 & 16.21 & 30.17 & 0.963 \\
\hline
\end{tabular}


Appendix (continued)

\begin{tabular}{|c|c|c|c|c|c|c|c|c|c|c|c|}
\hline Hole & Core 5 & & $\begin{array}{l}\text { Depth } \\
\text { (cm) }\end{array}$ & $\begin{array}{c}\text { SBD } \\
\text { (mbsf) }\end{array}$ & $\begin{array}{l}\text { MASS } \\
(\mathrm{g})\end{array}$ & $\underset{10^{-6} \mathrm{~m}^{3} / \mathrm{kg}}{X}$ & $\begin{array}{c}\mathrm{X}_{\mathrm{ARM}} \\
10^{-6 \mathrm{~m}^{3} / \mathrm{kg}}\end{array}$ & $\mathrm{X}_{\mathrm{ARM}} / \mathrm{X}$ & $\underset{(\mathrm{mA} / \mathrm{m} \mathrm{kg})}{\mathrm{SIRM}}$ & $\begin{array}{c}\text { HIRM } \\
(\mathrm{mA} / \mathrm{m} \mathrm{kg})\end{array}$ & $\mathrm{S}$ \\
\hline $645 G$ & $1 \mathrm{H}$ & 4 & 0 & 4.50 & 10.01 & 57.98 & 3398.36 & 58.61 & 14.26 & 35.17 & 0.951 \\
\hline $645 G$ & 1H & 4 & 17 & 4.67 & 10.36 & 44.07 & 1405.56 & 31.89 & 9.14 & 16.28 & 0.963 \\
\hline $645 G$ & $1 \mathrm{H}$ & 4 & 23 & 4.73 & 10.04 & 53.00 & 1020.42 & 19.25 & 8.12 & 18.48 & 0.954 \\
\hline $645 G$ & $1 \mathrm{H}$ & 4 & 35 & 4.85 & 10.98 & 57.10 & 1349.53 & 23.64 & 9.69 & 19.48 & 0.956 \\
\hline $645 G$ & 1H & 4 & 44 & 4.94 & 10.04 & 62.50 & 1495.40 & 23.93 & 9.67 & 17.70 & 0.963 \\
\hline $645 G$ & IH & 4 & 53 & 5.03 & 9.18 & 221.02 & 11912.50 & 53.90 & 46.96 & 10.46 & 0.996 \\
\hline $645 G$ & 1H & 4 & 62 & 5.12 & 9.32 & 647.50 & 7932.90 & 12.25 & 47.76 & 103.33 & 0.960 \\
\hline $645 G$ & 1H & 4 & 74 & 5.24 & 5.71 & 31.33 & 1877.60 & 59.93 & 5.13 & 13.85 & 0.969 \\
\hline $645 G$ & 1H & 4 & 84 & 5.34 & 10.55 & 55.60 & 1438.11 & 25.86 & 10.14 & 18.70 & 0.961 \\
\hline $645 G$ & 1H & 4 & 103 & 5.53 & 11.04 & 63.32 & 1503.67 & 23.75 & 9.96 & 18.51 & 0.959 \\
\hline $645 G$ & 1H & 4 & 118 & 5.68 & 10.75 & 70.05 & 1789.41 & 25.55 & 11.24 & 22.23 & 0.957 \\
\hline $645 G$ & 1H & 4 & 125 & 5.75 & 11.18 & 58.36 & 1567.21 & 26.85 & 10.69 & 21.71 & 0.955 \\
\hline $645 G$ & 1H & 4 & 133 & 5.83 & 10.56 & 67.00 & 1528.36 & 22.81 & 10.55 & 18.93 & 0.962 \\
\hline $645 G$ & $1 \mathrm{H}$ & 4 & 142 & 5.92 & 9.60 & 62.64 & 1544.18 & 24.65 & 9.20 & 19.67 & 0.959 \\
\hline $645 G$ & $1 \mathrm{H}$ & 5 & 10 & 6.10 & 10.75 & 60.43 & 2074.79 & 34.33 & 12.17 & 22.38 & 0.960 \\
\hline $645 G$ & $1 \mathrm{H}$ & 5 & 36 & 6.36 & 8.75 & 92.16 & 6068.05 & 65.85 & 19.97 & 28.32 & 0.975 \\
\hline $645 B$ & $1 \mathrm{x}$ & 1 & 80 & 0.80 & 6.46 & 118.63 & 10729.54 & 90.45 & 23.16 & 14.94 & 0.992 \\
\hline $645 B$ & $1 \mathrm{x}$ & 2 & 48 & 1.98 & 5.31 & 78.07 & 11098.10 & 142.15 & 23.99 & 56.80 & 0.975 \\
\hline $645 B$ & $1 x$ & 3 & 29 & 3.29 & 9.74 & 85.13 & 3493.98 & 41.04 & 13.19 & 29.33 & 0.957 \\
\hline $645 B$ & $3 x$ & 1 & 28 & 13.78 & 8.81 & 71.30 & 3985.88 & 55.90 & 11.63 & 14.79 & 0.978 \\
\hline $645 B$ & $3 x$ & 2 & 90 & 15.90 & 7.68 & 76.88 & 2831.70 & 36.83 & 8.93 & 23.73 & 0.959 \\
\hline $645 B$ & $4 x$ & 1 & 29 & 23.09 & 8.55 & 80.81 & 7151.69 & 88.50 & 20.01 & 19.27 & 0.984 \\
\hline $645 B$ & $5 x$ & 1 & 37 & 32.57 & 7.41 & 66.12 & 4491.42 & 67.93 & 11.77 & 36.95 & 0.953 \\
\hline $645 B$ & $5 x$ & 2 & 104 & 34.74 & 6.58 & 116.46 & 3408.95 & 29.27 & 8.78 & 16.92 & 0.975 \\
\hline $645 B$ & $5 x$ & 3 & 55 & 35.75 & 8.56 & 60.17 & 3665.96 & 60.92 & 9.15 & 24.20 & 0.955 \\
\hline $645 B$ & $6 x$ & 1 & 54 & 43.14 & 9.03 & 73.74 & 3919.24 & 53.15 & 11.30 & 18.24 & 0.971 \\
\hline $645 B$ & $8 x$ & 2 & 27 & 63.77 & 6.77 & 94.64 & 3016.00 & 31.87 & 8.89 & 14.76 & 0.978 \\
\hline $645 B$ & $9 x$ & 1 & 61 & 72.21 & 9.08 & 92.70 & 4143.93 & 44.70 & 12.55 & 25.48 & 0.963 \\
\hline $645 B$ & $9 x$ & 2 & 68 & 73.78 & 9.61 & 67.98 & 2609.43 & 38.39 & 8.90 & 11.52 & 0.975 \\
\hline $645 B$ & $9 \mathrm{X}$ & 3 & 55 & 75.15 & 9.31 & 126.84 & 6710.65 & 52.91 & 19.60 & 8.45 & 0.992 \\
\hline $645 B$ & $10 x$ & 1 & 104 & 82.34 & 7.91 & 39.71 & 2081.04 & 52.41 & 4.38 & 20.02 & 0.928 \\
\hline $645 B$ & $10 x$ & 2 & 47 & 83.27 & 9.05 & 40.26 & 1693.55 & 42.07 & 4.66 & 14.20 & 0.945 \\
\hline $645 B$ & $10 x$ & 3 & 6 & 84.36 & 9.11 & 17.93 & 1548.49 & 86.38 & 6.09 & 28.50 & 0.915 \\
\hline $645 B$ & $11 \mathrm{x}$ & 1 & 53 & 91.43 & 8.50 & 104.94 & 10074.93 & 96.01 & 27.84 & 20.10 & 0.988 \\
\hline $645 B$ & $11 x$ & 3 & 130 & 95.20 & 7.88 & 84.50 & 11547.90 & 136.67 & 27.63 & 26.01 & 0.985 \\
\hline $645 B$ & $11 x$ & 4 & 14 & 95.54 & 9.18 & 24.63 & 3003.31 & 121.92 & 10.32 & 17.38 & 0.969 \\
\hline $645 B$ & $13 x$ & 1 & 56 & 110.86 & 6.68 & 54.54 & 5854.31 & 107.34 & 31.91 & 52.06 & 0.978 \\
\hline $645 B$ & $13 x$ & 2 & 139 & 113.19 & 7.79 & 124.18 & 7433.03 & 59.86 & 21.11 & 43.06 & 0.968 \\
\hline $645 B$ & $13 x$ & 3 & 9 & 113.39 & 8.79 & 81.47 & 6843.09 & 84.00 & 17.30 & 26.75 & 0.973 \\
\hline $645 B$ & $13 x$ & 3 & 9 & 113.39 & 6.35 & 79.14 & 7345.39 & 92.82 & 12.97 & 28.58 & 0.972 \\
\hline $645 B$ & $13 x$ & 4 & 66 & 115.46 & 9.40 & 16.04 & 1193.87 & 74.44 & 3.34 & 15.32 & 0.914 \\
\hline $645 B$ & $14 x$ & 1 & 48 & 120.38 & 5.99 & 195.05 & 17654.21 & 90.51 & 28.91 & 7.05 & 0.997 \\
\hline $645 B$ & $14 x$ & 2 & 74 & 122.14 & 7.26 & 50.18 & 2839.61 & 56.59 & 7.77 & 30.37 & 0.943 \\
\hline $645 B$ & $14 x$ & 3 & 101 & 123.91 & 8.41 & 25.39 & 84.25 & 3.32 & 0.14 & 0.72 & 0.916 \\
\hline $645 B$ & $15 X$ & 1 & 68 & 130.18 & 9.54 & 51.36 & 2123.43 & 41.35 & 8.23 & 5.41 & 0.987 \\
\hline $645 B$ & $17 x$ & 2 & 34 & 150.64 & 8.32 & 15.10 & 2356.58 & 156.07 & 17.78 & 169.22 & 0.842 \\
\hline $645 B$ & $17 x$ & 3 & 10 & 151.90 & 8.19 & 7.67 & 376.88 & 49.14 & 7.04 & 69.11 & 0.839 \\
\hline $645 B$ & $17 x$ & 4 & 74 & 154.04 & 9.06 & 13.87 & 1305.51 & 94.15 & 17.49 & 2.54 & 0.997 \\
\hline $645 B$ & $18 x$ & 2 & 9 & 159.99 & 10.24 & 17.18 & 311.37 & 18.13 & 3.06 & 4.04 & 0.973 \\
\hline 645B & $19 \mathrm{X}$ & 2 & 60 & 170.20 & 9.46 & 38.51 & 3909.74 & 101.52 & 12.28 & 16.05 & 0.975 \\
\hline $645 B$ & $19 x$ & 3 & 107 & 172.17 & 9.20 & 31.41 & 2688.44 & 85.60 & 9.09 & 16.38 & 0.967 \\
\hline $645 B$ & $19 x$ & 5 & 102 & 175.12 & 9.26 & 39.34 & 1860.68 & 47.29 & 15.45 & 4.87 & 0.994 \\
\hline $645 B$ & $20 x$ & 1 & 114 & 178.24 & 8.58 & 38.07 & 2708.91 & 71.16 & 8.01 & 9.50 & 0.980 \\
\hline $645 B$ & $20 x$ & 3 & 109 & 181.19 & 8.82 & 68.37 & 4640.98 & 67.88 & 29.84 & 10.09 & 0.994 \\
\hline $645 B$ & $21 X$ & 4 & 87 & 192.77 & 8.52 & 32.44 & 1413.91 & 43.59 & 11.34 & 2.81 & 0.996 \\
\hline $645 B$ & $21 x$ & 5 & 120 & 194.60 & 9.43 & 26.64 & 1542.84 & 57.90 & 6.83 & 5.84 & 0.984 \\
\hline $645 B$ & $22 \mathrm{X}$ & 2 & 131 & 199.61 & 9.00 & 114.46 & 8006.14 & 69.95 & 24.76 & 27.63 & 0.980 \\
\hline
\end{tabular}


Appendix (continued)

\begin{tabular}{|c|c|c|c|c|c|c|c|c|c|c|c|}
\hline Hole & Core S & & $\begin{array}{l}\text { Depth } \\
\text { (cm) }\end{array}$ & $\begin{array}{c}\text { SBD } \\
\text { (mbsf) }\end{array}$ & $\begin{array}{c}\text { MASS } \\
(\mathrm{g})\end{array}$ & $\underset{10^{-6} \mathrm{~m}^{3} / \mathrm{kg}}{X}$ & $\underset{10-6 \mathrm{~m}^{3} / \mathrm{kg}}{\mathrm{X}_{\mathrm{ARM}}}$ & $x_{\text {ARM }} / \mathrm{X}$ & $\underset{(\mathrm{mA} / \mathrm{m} \mathrm{kg})}{\mathrm{SIRM}}$ & $\begin{array}{c}\text { HIRM } \\
(\mathrm{mA} / \mathrm{m} \mathrm{kg})\end{array}$ & S \\
\hline $645 B$ & $22 \mathrm{x}$ & 3 & 92 & 200.72 & 9.76 & 90.10 & 18437.47 & 204.63 & 254.44 & 361.22 & 0.972 \\
\hline $645 B$ & $22 \mathrm{x}$ & 4 & 57 & 201.87 & 8.56 & 49.90 & 2951.38 & 59.15 & 33.91 & 12.86 & 0.994 \\
\hline $645 B$ & $22 \mathrm{x}$ & 6 & 104 & 205.34 & 8.61 & 194.06 & 11708.95 & 60.34 & 32.65 & 19.92 & 0.989 \\
\hline $645 B$ & $23 x$ & 1 & 135 & 207.95 & 6.46 & 157.52 & 13825.51 & 87.77 & 37.55 & 8.80 & 0.997 \\
\hline $645 B$ & $23 \mathrm{x}$ & 2 & 52 & 208.62 & 8.00 & 43.97 & 1738.07 & 39.53 & 8.81 & 17.24 & 0.969 \\
\hline $645 B$ & $23 x$ & 3 & 37 & 209.97 & 8.62 & 53.92 & 3219.26 & 59.70 & 11.47 & 8.37 & 0.987 \\
\hline $645 B$ & $23 x$ & 4 & 105 & 212.15 & 7.15 & 5.27 & 389.18 & 73.83 & 1.21 & 1.51 & 0.982 \\
\hline $645 B$ & $24 \mathrm{X}$ & 1 & 71 & 216.91 & 8.10 & 1.55 & 482.35 & 311.00 & 1.16 & 1.46 & 0.980 \\
\hline $645 B$ & $24 \mathrm{x}$ & 2 & 44 & 218.14 & 7.67 & 1.64 & 550.01 & 335.80 & 1.25 & 0.93 & 0.989 \\
\hline $645 B$ & $24 \mathrm{X}$ & 3 & 106 & 220.26 & 7.51 & 1.67 & 523.92 & 313.20 & 0.72 & 3.11 & 0.935 \\
\hline $645 B$ & $24 \mathrm{X}$ & 4 & 18 & 220.88 & 7.69 & 1.63 & 525.55 & 321.70 & 1.21 & 1.46 & 0.982 \\
\hline $645 B$ & $24 \mathrm{X}$ & 5 & 36 & 222.56 & 8.30 & 1.51 & 417.75 & 276.00 & 1.01 & 1.60 & 0.974 \\
\hline $645 B$ & $24 \mathrm{X}$ & 5 & 36 & 222.56 & 5.40 & 2.33 & 425.97 & 183.10 & 0.75 & 0.19 & 0.997 \\
\hline $645 B$ & $26 \mathrm{X}$ & 1 & 69 & 236.29 & 7.29 & 8.62 & 674.50 & 78.28 & 2.62 & 8.27 & 0.954 \\
\hline $645 B$ & $26 \mathrm{x}$ & 2 & 102 & 238.12 & 7.27 & 60.48 & 1010.55 & 16.71 & 4.02 & 17.76 & 0.936 \\
\hline $645 B$ & $26 x$ & 3 & 87 & 239.47 & 7.77 & 45.27 & 1593.72 & 35.20 & 9.68 & 6.16 & 0.990 \\
\hline $645 B$ & $26 \mathrm{X}$ & 4 & 86 & 240.96 & 8.72 & 10.08 & 888.19 & 88.07 & 6.90 & 3.58 & 0.991 \\
\hline $645 B$ & $26 \mathrm{X}$ & 5 & 103 & 242.63 & 7.86 & 41.56 & 1896.41 & 45.63 & 10.11 & 15.24 & 0.976 \\
\hline $645 B$ & $26 \mathrm{X}$ & 6 & 81 & 243.91 & 9.13 & 71.55 & 2110.36 & 29.49 & 10.65 & 24.04 & 0.959 \\
\hline $645 B$ & $26 x$ & 7 & 27 & 244.87 & 8.42 & 53.71 & 1505.75 & 28.03 & 6.58 & 12.25 & 0.969 \\
\hline $645 B$ & $27 x$ & 1 & 124 & 246.44 & 8.71 & 24.52 & 534.82 & 21.81 & 1.95 & 7.36 & 0.934 \\
\hline $645 B$ & $27 \mathrm{X}$ & 2 & 33 & 247.03 & 7.65 & 59.12 & 1006.34 & 17.02 & 4.40 & 9.67 & 0.966 \\
\hline $645 B$ & $27 x$ & 3 & 130 & 249.50 & 8.43 & 125.18 & 4716.05 & 37.67 & 25.80 & 23.14 & 0.985 \\
\hline $645 B$ & $27 \mathrm{X}$ & 5 & 118 & 252.38 & 8.46 & 98.01 & 2370.30 & 24.18 & 19.73 & 1.17 & 0.999 \\
\hline $645 B$ & $27 X$ & 6 & 39 & 253.09 & 7.84 & 64.10 & 1945.95 & 30.36 & 11.76 & 9.68 & 0.987 \\
\hline $645 B$ & $28 \mathrm{X}$ & 1 & 98 & 255.88 & 7.53 & 66.73 & 772.96 & 11.58 & 4.09 & 4.25 & 0.984 \\
\hline $645 B$ & $28 \mathrm{X}$ & 2 & 76 & 257.16 & 8.20 & 26.04 & 2071.79 & 79.55 & 10.19 & 16.31 & 0.974 \\
\hline $645 B$ & $28 \mathrm{X}$ & 3 & 54 & 258.44 & 7.38 & 78.30 & 1967.83 & 25.13 & 12.14 & 14.23 & 0.983 \\
\hline $645 B$ & $28 \mathrm{X}$ & 3 & 54 & 258.44 & 5.80 & 86.64 & 1913.66 & 22.09 & 9.33 & 16.29 & 0.980 \\
\hline $645 B$ & $28 \mathrm{X}$ & 4 & 54 & 259.94 & 8.82 & 31.34 & 2874.78 & 91.74 & 18.37 & 9.82 & 0.991 \\
\hline $645 B$ & $29 x$ & 1 & 39 & 264.99 & 8.14 & 57.10 & 1190.38 & 20.85 & 6.48 & 13.66 & 0.966 \\
\hline 645D & IR & 1 & 105 & 266.75 & 8.50 & 39.91 & 2227.76 & 55.83 & 24.58 & 16.60 & 0.989 \\
\hline $645 B$ & $29 \mathrm{X}$ & 2 & 108 & 267.18 & 9.46 & 27.89 & 869.97 & 31.20 & 6.18 & 5.51 & 0.983 \\
\hline 645D & 1R & 2 & 99 & 268.19 & 7.20 & 13.96 & 1052.14 & 75.38 & 5.00 & 3.41 & 0.990 \\
\hline $645 B$ & $29 X$ & 3 & 63 & 268.23 & 8.65 & 55.19 & 2781.97 & 50.41 & 29.46 & 53.87 & 0.968 \\
\hline 645D & IR & 3 & 23 & 268.93 & 7.65 & 31.20 & 1442.51 & 46.23 & 7.95 & 15.81 & 0.970 \\
\hline $645 B$ & $29 x$ & 4 & 27 & 269.37 & 7.25 & 24.26 & 457.63 & 18.86 & 1.24 & 2.37 & 0.972 \\
\hline 645D & $1 \mathbf{R}$ & 4 & 76 & 270.96 & 7.64 & 13.15 & 620.58 & 47.18 & 1.48 & 9.60 & 0.901 \\
\hline $645 B$ & $29 x$ & 5 & 86 & 271.46 & 8.22 & 27.51 & 842.87 & 30.64 & 4.10 & 2.04 & 0.992 \\
\hline $645 D$ & $2 R$ & 1 & 82 & 273.72 & 6.11 & 8.22 & 595.65 & 72.43 & 1.26 & 14.89 & 0.855 \\
\hline $645 D$ & $2 R$ & 2 & 53 & 274.93 & 7.58 & 6.63 & 544.44 & 82.13 & 1.09 & 4.84 & 0.933 \\
\hline $645 D$ & $2 R$ & 3 & 44 & 276.34 & 6.57 & 5.74 & 464.27 & 80.93 & 0.80 & 5.45 & 0.910 \\
\hline 645D & $2 R$ & 4 & 121 & 278.61 & 8.35 & 7.52 & 486.72 & 64.70 & 0.89 & 3.47 & 0.935 \\
\hline $645 \mathrm{D}$ & $2 R$ & 6 & 19 & 280.59 & 6.37 & 5.92 & 462.08 & 78.10 & 0.64 & 2.87 & 0.942 \\
\hline $645 B$ & $31 x$ & 4 & 85 & 289.15 & 6.69 & 73.24 & 8276.06 & 113.01 & 19.72 & 33.29 & 0.977 \\
\hline $645 B$ & $31 x$ & 5 & 138 & 291.18 & 8.75 & 96.20 & 7234.46 & 75.21 & 41.17 & 70.53 & 0.970 \\
\hline $645 B$ & $31 x$ & 5 & 138 & 291.18 & 5.47 & 89.57 & 6155.78 & 68.73 & 17.59 & 42.65 & 0.973 \\
\hline $645 B$ & $30 x$ & 6 & 60 & 291.70 & 7.44 & 13.51 & 608.05 & 45.01 & 0.99 & 3.13 & 0.953 \\
\hline $645 B$ & $30 x$ & 6 & 60 & 291.70 & 5.55 & 18.11 & 588.08 & 32.48 & 0.75 & 3.56 & 0.947 \\
\hline $645 B$ & $32 X$ & 1 & 114 & 294.64 & 8.67 & 110.12 & 12383.28 & 112.45 & 54.10 & 181.42 & 0.942 \\
\hline $645 B$ & $32 \mathrm{X}$ & 3 & 123 & 297.73 & 8.21 & 82.63 & 8073.41 & 97.71 & 40.88 & 110.49 & 0.956 \\
\hline $645 B$ & $32 \mathrm{x}$ & 4 & 38 & 298.38 & 8.52 & 84.05 & 6909.55 & 82.21 & 44.18 & 34.40 & 0.987 \\
\hline $645 B$ & $32 \mathrm{x}$ & 5 & 17 & 299.67 & 7.47 & 63.91 & 6162.67 & 96.43 & 20.18 & 50.90 & 0.962 \\
\hline $645 B$ & $32 \mathrm{X}$ & 7 & 19 & 302.69 & 8.92 & 92.95 & 1971.46 & 21.21 & 28.17 & 203.45 & 0.871 \\
\hline $645 E$ & $2 R$ & 1 & 49 & 311.29 & 7.59 & 86.07 & 2307.65 & 26.81 & 12.60 & 10.40 & 0.987 \\
\hline $645 \mathrm{D}$ & $6 R$ & 1 & 28 & 311.68 & 8.70 & 67.87 & 4106.45 & 60.51 & 24.04 & 46.56 & 0.966 \\
\hline $645 \mathrm{D}$ & $8 R$ & 1 & 37 & 340.77 & 7.82 & 41.77 & 1345.92 & 32.22 & 7.35 & 25.65 & 0.945 \\
\hline
\end{tabular}


Appendix (continued)

\begin{tabular}{|c|c|c|c|c|c|c|c|c|c|c|c|}
\hline Hole & Core S & & $\begin{array}{l}\text { Depth } \\
\text { (cm) }\end{array}$ & $\begin{array}{c}\text { SBD } \\
\text { (mbsf) }\end{array}$ & $\begin{array}{c}\text { MASS } \\
(\mathrm{g})\end{array}$ & $\underset{10^{-6} \mathrm{~m}^{3} / \mathrm{kg}}{\mathrm{X}}$ & $\underset{10^{-6 \mathrm{~m}^{3} / \mathrm{kg}}}{\mathrm{X}_{\mathrm{ARM}}}$ & $X_{\text {ARM }} / X$ & $\underset{(\mathrm{mA} / \mathrm{m} \mathrm{kg})}{\operatorname{SIRM}}$ & $\begin{array}{c}\text { HIRM } \\
(\mathrm{mA} / \mathrm{m} \mathrm{kg})\end{array}$ & $\mathrm{S}$ \\
\hline $645 D$ & $8 \mathrm{R}$ & 2 & 87 & 342.77 & 6.74 & 26.09 & 1427.76 & 54.71 & 7.74 & 24.69 & 0.957 \\
\hline $645 D$ & $8 \mathrm{R}$ & 3 & 69 & 344.09 & 7.28 & 13.81 & 942.56 & 68.27 & 5.62 & 10.34 & 0.973 \\
\hline $645 D$ & $9 \mathrm{R}$ & 4 & 58 & 355.08 & 8.72 & 18.73 & 1482.18 & 79.14 & 13.08 & 12.16 & 0.984 \\
\hline $645 D$ & $9 R$ & 5 & 27 & 356.27 & 8.71 & 17.31 & 1350.90 & 78.05 & 9.90 & 12.95 & 0.977 \\
\hline $645 \mathrm{D}$ & 9R & 6 & 15 & 357.65 & 6.98 & 12.60 & 547.87 & 43.49 & 2.26 & 3.13 & 0.981 \\
\hline $645 D$ & 10R: & 1 & 46 & 360.16 & 7.27 & 20.74 & 1425.28 & 68.73 & 8.01 & 12.25 & 0.978 \\
\hline 645D & 10R & 2 & 80 & 362.00 & 7.15 & 7.03 & 552.76 & 78.65 & 1.01 & 6.87 & 0.903 \\
\hline $645 \mathrm{D}$ & 10R & 3 & 49 & 363.19 & 6.74 & 11.18 & 450.32 & 40.27 & 0.58 & 1.90 & 0.956 \\
\hline 645D & 10R & 4 & 76 & 364.96 & 6.88 & 7.30 & 470.56 & 64.43 & 0.98 & 2.16 & 0.970 \\
\hline 645D & IIR & 1 & 81 & 370.21 & 7.19 & 8.74 & 468.09 & 53.58 & 0.77 & 2.29 & 0.957 \\
\hline 645D & IIR & 2 & 97 & 371.87 & 8.07 & 9.34 & 495.04 & 53.00 & 1.25 & 2.33 & 0.970 \\
\hline 645D & IIR & 3 & 75 & 373.15 & 7.53 & 8.34 & 590.77 & 70.82 & 1.00 & 3.96 & 0.941 \\
\hline 645D & IIR & 4 & 100 & 374.90 & 6.43 & 11.72 & 476.92 & 40.68 & 1.14 & 20.92 & 0.765 \\
\hline 645D & IIR & 5 & 83 & 376.23 & 8.20 & 12.26 & 578.66 & 47.21 & 1.39 & 7.89 & 0.907 \\
\hline 645D & IIR & 6 & 73 & 377.63 & 5.10 & 9.85 & 311.36 & 31.60 & 0.50 & 3.79 & 0.922 \\
\hline 645D & IIR & 7 & 36 & 378.76 & 6.60 & 15.23 & 391.35 & 25.70 & 0.85 & 5.46 & 0.916 \\
\hline 645D & $12 \mathrm{R}$ & 1 & 100 & 380.00 & 7.21 & 8.71 & 297.60 & 34.16 & 0.69 & 3.42 & 0.929 \\
\hline $645 \mathrm{D}$ & $13 R$ & 1 & 139 & 389.99 & 9.10 & 17.95 & 1065.22 & 59.35 & 7.31 & 11.82 & 0.971 \\
\hline 645D & $13 R$ & 2 & 55 & 390.65 & 8.78 & 14.31 & 519.11 & 36.28 & 1.71 & 16.57 & 0.830 \\
\hline 645D & $13 R$ & 3 & 104 & 392.64 & 8.47 & 8.90 & 473.59 & 53.22 & 1.29 & 5.79 & 0.924 \\
\hline $645 D$ & $13 R$ & 4 & 54 & 393.64 & 8.47 & 13.35 & 762.67 & 57.13 & 4.52 & 7.40 & 0.972 \\
\hline $645 D$ & $13 R$ & 5 & 43 & 395.03 & 9.47 & 13.27 & 702.43 & 52.95 & 5.95 & 0.25 & 0.999 \\
\hline $645 D$ & $13 R$ & 6 & 69 & 396.79 & 9.60 & 10.47 & 405.54 & 38.74 & 1.51 & 8.67 & 0.890 \\
\hline $645 D$ & $14 R$ & 1 & 66 & 398.96 & 8.43 & 25.33 & 3141.30 & 123.99 & 36.87 & 52.00 & 0.976 \\
\hline $645 D$ & $14 R$ & 2 & 46 & 400.26 & 9.02 & 20.89 & 2051.14 & 98.18 & 29.15 & 37.40 & 0.977 \\
\hline 645D & $14 \mathrm{R}$ & 3 & 127 & 402.57 & 8.45 & 11.89 & 769.38 & 64.69 & 4.34 & 6.72 & 0.974 \\
\hline $645 \mathrm{D}$ & $14 \mathrm{R}$ & 4 & 28 & 403.08 & 8.02 & 26.63 & 2811.60 & 105.58 & 30.39 & 70.17 & 0.963 \\
\hline 645D & $14 \mathrm{R}$ & 5 & 41 & 404.71 & 8.43 & 37.26 & 5554.61 & 149.09 & 101.51 & 520.02 & 0.914 \\
\hline 645D & $14 \mathrm{R}$ & 5 & 41 & 404.71 & 7.73 & 40.63 & 5058.28 & 124.50 & 76.74 & 239.28 & 0.952 \\
\hline 645D & $15 R$ & 1 & 140 & 409.30 & 8.55 & 27.92 & 4232.57 & 151.61 & 61.02 & 275.80 & 0.923 \\
\hline 645D & $15 R$ & 2 & 112 & 410.52 & 7.79 & 20.96 & 1104.04 & 52.66 & 3.67 & 11.79 & 0.950 \\
\hline 645D & $15 \mathrm{R}$ & 3 & 34 & 411.24 & 8.82 & 21.37 & 1687.01 & 78.96 & 14.69 & 23.63 & 0.972 \\
\hline $645 \mathrm{D}$ & $15 R$ & 4 & 139 & 413.79 & 7.97 & 20.49 & 1787.48 & 87.23 & 17.44 & 21.25 & 0.981 \\
\hline 645D & $15 R$ & 5 & 132 & 415.22 & 8.08 & 18.66 & 1968.07 & 105.48 & 25.95 & 102.66 & 0.936 \\
\hline 645D & $15 R$ & 6 & 139 & 416.79 & 8.58 & 33.68 & 1780.90 & 52.88 & 16.08 & 25.06 & 0.973 \\
\hline 645D & $16 R$ & 1 & 59 & 418.19 & 8.60 & 17.53 & 861.87 & 49.17 & 3.75 & 19.94 & 0.909 \\
\hline 645D & $16 \mathrm{R}$ & 2 & 29 & 419.39 & 6.72 & 11.22 & 792.09 & 70.62 & 4.34 & 5.86 & 0.982 \\
\hline 645D & $16 R$ & 3 & 107 & 421.67 & 7.86 & 108.69 & 11299.02 & 103.96 & 109.14 & 248.37 & 0.964 \\
\hline 645D & $17 R$ & 2 & 142 & 430.12 & 6.28 & 18.00 & 971.82 & 53.98 & 3.10 & 17.45 & 0.929 \\
\hline 645D & 17R & 3 & 88 & 431.08 & 6.89 & 29.17 & 4028.31 & 138.08 & 40.76 & 497.71 & 0.832 \\
\hline 645D & $17 R$ & 4 & 25 & 431.95 & 7.42 & 23.70 & 2616.18 & 110.37 & 35.03 & 190.82 & 0.919 \\
\hline 645D & $17 R$ & 5 & 134 & 434.54 & 6.48 & 31.02 & 3173.66 & 102.31 & 20.74 & 1.23 & 0.999 \\
\hline 645D & $17 R$ & 7 & 102 & 437.22 & 8.70 & 88.08 & 21938.43 & 249.06 & 345.93 & 642.14 & 0.968 \\
\hline $645 \mathrm{D}$ & $18 R$ & 1 & 65 & 437.55 & 8.87 & 123.22 & 9612.61 & 78.01 & 44.53 & 21.51 & 0.991 \\
\hline $645 \mathrm{D}$ & $18 R$ & 3 & 24 & 440.14 & 7.47 & 20.18 & 1701.45 & 84.31 & 12.07 & 13.05 & 0.984 \\
\hline 645D & $19 R$ & 1 & 21 & 446.71 & 6.54 & 86.44 & 18270.25 & 211.36 & 238.51 & 2148.39 & 0.882 \\
\hline 645D & $19 R$ & 2 & 18 & 448.18 & 10.02 & 21.31 & 1577.62 & 74.02 & 17.85 & 6.16 & 0.993 \\
\hline 645D & $19 R$ & 3 & 106 & 450.56 & 8.48 & 117.04 & 8461.38 & 72.30 & 26.02 & 44.04 & 0.971 \\
\hline $645 \mathrm{D}$ & $19 R$ & 4 & 104 & 452.04 & 8.21 & 18.36 & 1124.07 & 61.22 & 2.81 & 19.76 & 0.884 \\
\hline 645D & $19 R$ & 5 & 111 & 453.61 & 8.78 & 20.03 & 1353.01 & 67.54 & 13.53 & 30.41 & 0.961 \\
\hline $645 E$ & $4 R$ & 1 & 130 & 456.50 & 7.30 & 25.81 & 1073.00 & 41.57 & 6.08 & 16.36 & 0.961 \\
\hline $645 D$ & $20 R$ & 1 & 65 & 456.85 & 8.18 & 38.39 & 3614.65 & 94.14 & 34.83 & 39.70 & 0.981 \\
\hline $645 E$ & $4 R$ & 2 & 137 & 458.07 & 7.85 & 24.01 & 655.99 & 27.33 & 2.21 & 17.67 & 0.875 \\
\hline $645 D$ & $20 R$ & 2 & 85 & 458.55 & 8.67 & 14.49 & 686.83 & 47.40 & 4.61 & 9.22 & 0.965 \\
\hline $645 E$ & $4 \mathrm{R}$ & 3 & 106 & 459.26 & 6.76 & 29.73 & 1775.52 & 59.71 & 18.84 & 5.30 & 0.996 \\
\hline 645D & $20 R$ & 5 & 116 & 463.36 & 8.62 & 5.83 & 255.05 & 43.75 & 0.39 & 1.06 & 0.954 \\
\hline $645 E$ & $6 R$ & 1 & 112 & 475.62 & 7.32 & 17.16 & 523.11 & 30.48 & 0.57 & 1.44 & 0.963 \\
\hline
\end{tabular}


Appendix (continued)

\begin{tabular}{|c|c|c|c|c|c|c|c|c|c|c|c|}
\hline Hole & Core & & $\begin{array}{l}\text { Depth } \\
\text { (cm) }\end{array}$ & $\begin{array}{c}\text { SBD } \\
\text { (mbsf) }\end{array}$ & $\begin{array}{c}\text { MASS } \\
(\mathrm{g})\end{array}$ & $\underset{10-6 \mathrm{~m}^{3} / \mathrm{kg}}{\mathrm{X}}$ & $\underset{10-6 \mathrm{~m}^{3} / \mathrm{kg}}{\mathrm{X}_{\mathrm{ARM}}}$ & $\mathrm{X}_{\mathrm{ARM}} / \mathrm{X}$ & $\begin{array}{c}\text { SIRM } \\
(\mathrm{mA} / \mathrm{m} \mathrm{kg})\end{array}$ & $\begin{array}{c}\underset{(\mathrm{mA} / \mathrm{m} \mathrm{kg})}{\mathrm{HIRM}} \\
\text { (1) }\end{array}$ & $\mathrm{S}$ \\
\hline $645 E$ & $6 \mathrm{R}$ & 5 & 105 & 481.55 & 8.26 & 19.77 & 1152.25 & 58.28 & 7.96 & 1.31 & 0.997 \\
\hline $645 E$ & $7 R$ & 3 & 90 & 488.00 & 7.14 & 8.80 & 450.43 & 51.20 & 0.89 & 1.56 & 0.975 \\
\hline $645 E$ & $7 R$ & 4 & 95 & 489.55 & 7.42 & 8.47 & 379.59 & 44.84 & 0.58 & 1.38 & 0.965 \\
\hline $645 E$ & $7 R$ & 5 & 98 & 491.08 & 7.05 & 8.91 & 406.82 & 45.66 & 0.51 & 1.61 & 0.956 \\
\hline $645 E$ & $8 R$ & 1 & 13 & 493.83 & 6.82 & 11.05 & 551.14 & 49.87 & 1.56 & 0.07 & 0.999 \\
\hline $645 E$ & $8 R$ & 2 & 38 & 495.58 & 7.15 & 12.30 & 381.63 & 31.03 & 1.04 & 1.06 & 0.985 \\
\hline $645 E$ & $8 R$ & 3 & 60 & 497.30 & 7.77 & 12.93 & 357.97 & 27.68 & 0.54 & 1.51 & 0.956 \\
\hline $645 E$ & $8 R$ & 5 & 70 & 500.40 & 7.16 & 10.53 & 433.03 & 41.13 & 0.54 & 1.35 & 0.964 \\
\hline $645 E$ & $9 \mathrm{R}$ & 1 & 21 & 503.61 & 5.95 & 10.56 & 343.52 & 32.54 & 0.33 & 1.19 & 0.958 \\
\hline $645 E$ & $9 R$ & 2 & 48 & 505.38 & 5.87 & 6.42 & 368.54 & 57.40 & 0.38 & 0.85 & 0.974 \\
\hline $645 E$ & $9 \mathrm{R}$ & 3 & 83 & 507.23 & 6.37 & 7.89 & 431.12 & 54.65 & 0.48 & 1.98 & 0.948 \\
\hline $645 \mathrm{E}$ & 9R & 5 & 102 & 510.42 & 6.23 & 10.08 & 437.98 & 43.44 & 0.89 & 0.85 & 0.988 \\
\hline $645 E$ & $9 \mathrm{R}$ & 6 & 97 & 511.87 & 5.49 & 6.86 & 363.61 & 52.97 & 0.35 & 1.59 & 0.950 \\
\hline $645 E$ & $10 R$ & 2 & 5 & 514.55 & 18.67 & 10.16 & 399.63 & 39.33 & 5.03 & 1.01 & 0.992 \\
\hline $645 \mathrm{E}$ & IIR & 1 & 130 & 523.90 & 18.68 & 16.68 & 409.57 & 24.56 & 4.55 & 25.03 & 0.794 \\
\hline $645 E$ & $11 R$ & 2 & 81 & 524.91 & 20.95 & 18.89 & 1276.07 & 67.56 & 35.54 & 13.31 & 0.984 \\
\hline $645 E$ & IIR & 3 & 143 & 527.03 & 14.47 & 13.46 & 576.05 & 42.81 & 5.70 & 13.82 & 0.930 \\
\hline $645 E$ & $13 R$ & 2 & 130 & 544.60 & 9.08 & 9.68 & 295.25 & 30.49 & 0.45 & 0.85 & 0.966 \\
\hline $645 \mathrm{E}$ & $13 R$ & 4 & 36 & 546.66 & 13.94 & 19.20 & 1705.89 & 88.87 & 33.18 & 27.12 & 0.977 \\
\hline $645 E$ & $13 R$ & 6 & 67 & 549.97 & 18.36 & 51.25 & 6320.48 & 123.33 & 334.58 & 1153.04 & 0.873 \\
\hline $645 \mathrm{E}$ & $14 R$ & 4 & 76 & 556.76 & 7.59 & 26.65 & 983.01 & 36.89 & 4.80 & 8.40 & 0.973 \\
\hline $645 E$ & $14 R$ & 7 & 17 & 560.67 & 7.48 & 11.76 & 296.44 & 25.21 & 0.42 & 1.09 & 0.961 \\
\hline $645 \mathrm{E}$ & $15 R$ & 2 & 91 & 563.51 & 8.63 & 64.05 & 5005.04 & 78.14 & 69.34 & 47.86 & 0.988 \\
\hline $645 E$ & $16 R$ & 1 & 9 & 570.99 & 7.69 & 8.17 & 375.25 & 45.94 & 0.50 & 1.73 & 0.947 \\
\hline $645 E$ & $16 R$ & 2 & 65 & 573.05 & 7.75 & 14.59 & 466.69 & 31.99 & 0.60 & 1.24 & 0.968 \\
\hline $645 \mathrm{E}$ & $16 R$ & 3 & 24 & 574.14 & 7.42 & 13.54 & 398.73 & 29.44 & 0.60 & 0.77 & 0.981 \\
\hline $645 E$ & $16 R$ & 5 & 131 & 578.21 & 7.78 & 12.92 & 424.52 & 32.86 & 0.53 & 1.42 & 0.959 \\
\hline $645 \mathrm{E}$ & $17 R$ & 1 & 38 & 580.88 & 7.94 & 17.40 & 224.04 & 12.87 & 0.35 & 0.91 & 0.958 \\
\hline $645 \mathrm{E}$ & $17 R$ & 3 & 93 & 584.43 & 7.49 & 26.84 & 443.47 & 16.53 & 0.93 & 7.20 & 0.884 \\
\hline $645 \mathrm{E}$ & $17 R$ & 5 & 49 & 586.99 & 7.63 & 9.88 & 282.87 & 28.63 & 0.43 & 1.15 & 0.960 \\
\hline $645 \mathrm{E}$ & $17 R$ & 6 & 59 & 588.59 & 7.24 & 10.41 & 324.48 & 31.17 & 0.48 & 1.74 & 0.948 \\
\hline $645 \mathrm{E}$ & $18 R$ & 1 & 118 & 591.28 & 6.63 & 24.63 & 313.60 & 12.73 & 0.44 & 1.37 & 0.958 \\
\hline $645 E$ & $18 R$ & 2 & 37 & 591.97 & 6.69 & 20.66 & 274.17 & 13.27 & 0.36 & 2.03 & 0.925 \\
\hline $645 \mathrm{E}$ & $19 R$ & 1 & 103 & 600.83 & 9.49 & 22.50 & 419.11 & 18.62 & 0.94 & 2.76 & 0.944 \\
\hline $645 \mathrm{E}$ & $19 R$ & 2 & 138 & 602.68 & 8.06 & 21.82 & 267.00 & 12.24 & 0.65 & 8.24 & 0.795 \\
\hline $645 \mathrm{E}$ & 20R & 1 & 112 & 610.42 & 8.42 & 17.90 & 1220.32 & 68.16 & 8.59 & 1.82 & 0.996 \\
\hline $645 E$ & $20 R$ & 2 & 101 & 611.81 & 7.89 & 11.15 & 307.62 & 27.60 & 0.71 & 5.58 & 0.876 \\
\hline $645 E$ & 20R & 3 & 56 & 612.86 & 8.69 & 14.46 & 651.85 & 45.09 & 1.51 & 2.43 & 0.972 \\
\hline $645 \mathrm{E}$ & $23 R$ & 1 & 22 & 628.62 & 7.55 & 24.96 & 1359.61 & 54.47 & 9.50 & 0.78 & 0.999 \\
\hline $645 E$ & $23 R$ & 3 & 117 & 632.57 & 7.91 & 31.76 & 378.79 & 11.93 & 0.70 & 3.30 & 0.926 \\
\hline $645 \mathrm{E}$ & $24 R$ & 1 & 18 & 638.18 & 7.75 & 32.42 & 1212.51 & 37.40 & 3.91 & 9.81 & 0.961 \\
\hline $645 E$ & $24 R$ & 2 & 17 & 639.67 & 18.53 & 15.12 & 431.05 & 28.51 & 2.19 & 2.00 & 0.966 \\
\hline $645 \mathrm{E}$ & $25 R$ & 1 & 70 & 648.40 & 18.64 & 12.40 & 309.02 & 24.92 & 1.39 & 1.69 & 0.955 \\
\hline $645 \mathrm{E}$ & $25 R$ & 2 & 121 & 650.41 & 8.08 & 10.88 & 539.21 & 49.54 & 0.91 & 0.46 & 0.992 \\
\hline $645 \mathrm{E}$ & $26 R$ & 1 & 144 & 658.54 & 19.12 & 12.35 & 359.74 & 29.12 & 1.67 & 1.46 & 0.967 \\
\hline $645 \mathrm{E}$ & 26R & 2 & 120 & 659.80 & 21.05 & 12.83 & 349.07 & 27.20 & 1.79 & 1.51 & 0.964 \\
\hline $645 E$ & 26R & 3 & 127 & 661.37 & 17.94 & 13.80 & 451.18 & 32.71 & 1.79 & 1.88 & 0.962 \\
\hline $645 E$ & $27 R$ & 2 & 145 & 669.65 & 18.95 & 11.87 & 388.62 & 32.75 & 1.53 & 2.19 & 0.946 \\
\hline $645 E$ & $27 R$ & 3 & 145 & 671.15 & 18.96 & 12.26 & 465.94 & 38.01 & 3.23 & 5.41 & 0.936 \\
\hline $645 E$ & $27 R$ & 4 & 2 & 671.22 & 18.68 & 12.58 & 394.24 & 31.35 & 1.77 & 2.28 & 0.952 \\
\hline $645 E$ & $27 R$ & 6 & 2 & 674.22 & 20.81 & 13.28 & 262.48 & 19.76 & 1.78 & 0.87 & 0.980 \\
\hline $645 E$ & $28 R$ & 1 & 134 & 677.74 & 20.81 & 12.98 & 648.91 & 50.00 & 7.51 & 1.22 & 0.993 \\
\hline $645 E$ & $29 R$ & 2 & 136 & 688.86 & 8.44 & 14.88 & 377.18 & 25.34 & 1.08 & 7.37 & 0.885 \\
\hline $645 E$ & $29 R$ & 4 & 67 & 691.17 & 6.12 & 24.63 & 407.06 & 16.53 & 0.91 & 10.03 & 0.865 \\
\hline $645 E$ & $29 R$ & 6 & 75 & 694.25 & 8.11 & 13.94 & 491.67 & 35.27 & 1.54 & 3.81 & 0.960 \\
\hline $645 E$ & $30 R$ & 1 & 116 & 696.76 & 10.01 & 13.81 & 375.75 & 27.22 & 1.08 & 4.30 & 0.920 \\
\hline $645 E$ & $32 R$ & 3 & 119 & 713.89 & 8.94 & 18.27 & 368.03 & 20.15 & 1.81 & 7.48 & 0.926 \\
\hline
\end{tabular}




\begin{tabular}{|c|c|c|c|c|c|c|c|c|c|c|c|}
\hline Hole & Core & & $\begin{array}{l}\text { Depth } \\
\text { (cm) }\end{array}$ & $\begin{array}{c}\text { SBD } \\
\text { (mbsf) }\end{array}$ & $\begin{array}{c}\text { MASS } \\
(\mathrm{g})\end{array}$ & $\underset{10^{-6} \mathrm{~m}^{3 /} / \mathrm{kg}}{\mathrm{X}}$ & $\frac{\mathrm{X}_{\mathrm{ARM}}}{10-6 \mathrm{~m}^{3} / \mathrm{kg}}$ & $\mathrm{X}_{\mathrm{ARM}} / \mathrm{X}$ & $\begin{array}{c}\text { SIRM } \\
(\mathrm{mA} / \mathrm{m} \mathrm{kg})\end{array}$ & $\underset{(\mathrm{mA} / \mathrm{m} \mathrm{kg})}{\mathrm{HIRM}}$ & $\mathrm{s}$ \\
\hline $645 E$ & $32 R$ & 4 & 119 & 715.39 & 8.82 & 21.37 & 373.04 & 17.46 & 1.11 & 5.64 & 0.910 \\
\hline $645 E$ & $33 R$ & 1 & 121 & 715.91 & 9.17 & 15.07 & 396.20 & 26.29 & 1.17 & 4.46 & 0.930 \\
\hline $645 E$ & 33R & 4 & 77 & 719.97 & 6.94 & 43.44 & 3338.56 & 76.85 & 23.10 & 38.43 & 0.977 \\
\hline $645 E$ & $33 R$ & 5 & 92 & 721.62 & 9.05 & 12.49 & 369.39 & 29.57 & 0.87 & 3.60 & 0.925 \\
\hline $645 \mathrm{E}$ & $34 R$ & 1 & 130 & 725.70 & 8.52 & 14.75 & 401.66 & 27.24 & 0.72 & 1.69 & 0.960 \\
\hline $645 E$ & $34 R$ & 2 & 130 & 727.20 & 8.71 & 11.54 & 354.24 & 30.70 & 0.61 & 1.28 & 0.964 \\
\hline $645 \mathrm{E}$ & $34 R$ & 3 & 130 & 728.70 & 7.93 & 15.84 & 339.34 & 21.42 & 0.55 & 1.69 & 0.951 \\
\hline $645 E$ & $34 R$ & 4 & 40 & 729.30 & 9.51 & 9.25 & 310.83 & 33.61 & 0.62 & 1.12 & 0.966 \\
\hline $645 E$ & $35 R$ & 2 & 51 & 736.01 & 9.06 & 11.09 & 362.46 & 32.68 & 0.82 & 2.05 & 0.954 \\
\hline $645 E$ & $35 R$ & 3 & 110 & 738.10 & 7.27 & 12.10 & 352.86 & 29.17 & 0.49 & 1.21 & 0.964 \\
\hline $645 E$ & $35 R$ & 4 & 2 & 738.52 & 7.53 & 11.68 & 402.74 & 34.49 & 0.55 & 1.29 & 0.965 \\
\hline $645 E$ & $36 R$ & 1 & 111 & 744.81 & 11.97 & 10.50 & 320.63 & 30.55 & 0.79 & 1.30 & 0.960 \\
\hline $645 \mathrm{E}$ & $36 R$ & 2 & 123 & 746.43 & 13.94 & 11.36 & 306.95 & 27.03 & 0.97 & 1.89 & 0.946 \\
\hline $645 E$ & $38 R$ & 1 & 28 & 763.28 & 19.64 & 16.89 & 4895.60 & 289.91 & 159.90 & 60.03 & 0.985 \\
\hline $645 \mathrm{E}$ & $38 R$ & 2 & 81 & 765.31 & 20.20 & 13.18 & 358.23 & 27.17 & 2.54 & 0.71 & 0.989 \\
\hline $645 E$ & $38 R$ & 4 & 129 & 768.79 & 20.97 & 15.04 & 790.73 & 52.59 & 9.84 & 13.16 & 0.944 \\
\hline $645 E$ & $39 R$ & 5 & 74 & 779.44 & 20.70 & 40.54 & 808.57 & 19.94 & 11.73 & 3.91 & 0.986 \\
\hline $645 \mathrm{E}$ & $40 R$ & 1 & 28 & 782.58 & 20.39 & 11.83 & 340.41 & 28.78 & 1.90 & 2.43 & 0.948 \\
\hline $645 \mathrm{E}$ & $40 R$ & 3 & 53 & 785.83 & 21.52 & 10.92 & 285.76 & 26.18 & 1.67 & 0.59 & 0.985 \\
\hline $645 E$ & $41 R$ & 1 & 14 & 792.14 & 18.15 & 10.04 & 246.34 & 24.54 & 1.20 & 1.28 & 0.961 \\
\hline $645 \mathrm{E}$ & 4IR & 1 & 138 & 793.38 & 9.72 & 18.09 & 333.33 & 18.42 & 0.69 & 1.78 & 0.950 \\
\hline $645 \mathrm{E}$ & $41 R$ & 2 & 37 & 793.87 & 21.67 & 10.96 & 291.78 & 26.63 & 1.94 & 1.37 & 0.970 \\
\hline $645 E$ & $41 R$ & 5 & 113 & 799.13 & 21.36 & 10.12 & 263.84 & 26.08 & 1.55 & 1.15 & 0.968 \\
\hline $645 \mathrm{E}$ & $42 R$ & 2 & 54 & 803.64 & 9.34 & 16.14 & 323.35 & 20.03 & 0.69 & 0.89 & 0.976 \\
\hline $645 E$ & $42 R$ & 3 & 35 & 804.95 & 8.06 & 12.47 & 273.70 & 21.95 & 0.54 & 1.09 & 0.968 \\
\hline $645 \mathrm{E}$ & 42R & 5 & 131 & 808.91 & 7.86 & 148.64 & 9690.79 & 65.19 & 136.05 & 71.88 & 0.992 \\
\hline $645 E$ & $43 R$ & 1 & 114 & 812.34 & 20.85 & 9.28 & 251.08 & 27.06 & 1.43 & 2.07 & 0.940 \\
\hline $645 \mathrm{E}$ & $43 R$ & 2 & 79 & 813.49 & 21.29 & 10.03 & 278.34 & 27.75 & 1.66 & 2.57 & 0.934 \\
\hline $645 E$ & $43 R$ & 3 & 97 & 815.17 & 18.19 & 12.43 & 554.17 & 44.58 & 5.64 & 1.63 & 0.989 \\
\hline $645 E$ & $43 R$ & 4 & 140 & 817.10 & 20.89 & 9.98 & 293.59 & 29.41 & 1.57 & 1.64 & 0.957 \\
\hline $645 \mathrm{E}$ & $43 R$ & 5 & 109 & 818.29 & 20.15 & 11.04 & 307.06 & 27.82 & 1.87 & 3.10 & 0.933 \\
\hline $645 E$ & $43 R$ & 6 & 40 & 819.10 & 21.51 & 15.65 & 1264.22 & 80.77 & 21.63 & 1.04 & 0.998 \\
\hline $645 E$ & $44 R$ & 1 & 145 & 822.25 & 7.94 & 11.08 & 381.00 & 34.40 & 0.71 & 1.48 & 0.967 \\
\hline $645 E$ & $44 R$ & 2 & 147 & 823.77 & 7.38 & 11.92 & 336.37 & 28.23 & 0.60 & 1.16 & 0.971 \\
\hline $645 E$ & $44 R$ & 3 & 135 & 825.15 & 8.71 & 14.42 & 467.61 & 32.42 & 1.30 & 1.10 & 0.985 \\
\hline $645 E$ & $44 R$ & 5 & 113 & 827.93 & 9.38 & 10.71 & 304.70 & 28.44 & 0.69 & 1.39 & 0.962 \\
\hline $645 E$ & $44 R$ & 6 & 110 & 829.40 & 9.30 & 9.46 & 280.43 & 29.66 & 0.61 & 1.20 & 0.963 \\
\hline $645 \mathrm{E}$ & $45 R$ & 1 & 131 & 831.81 & 9.33 & 13.46 & 302.69 & 22.48 & 0.63 & 1.20 & 0.965 \\
\hline $645 E$ & $45 R$ & 2 & 131 & 833.31 & 7.84 & 9.61 & 274.17 & 28.52 & 0.51 & 1.06 & 0.967 \\
\hline $645 E$ & $45 R$ & 3 & 131 & 834.81 & 8.72 & 10.08 & 324.59 & 32.19 & 0.61 & 1.46 & 0.959 \\
\hline $645 E$ & $45 R$ & 5 & 75 & 837.25 & 7.64 & 9.87 & 274.77 & 27.85 & 0.44 & 1.18 & 0.959 \\
\hline $645 E$ & $46 R$ & 1 & 127 & 841.37 & 7.89 & 11.15 & 360.01 & 32.30 & 0.66 & 1.03 & 0.975 \\
\hline $645 E$ & $46 R$ & 2 & 127 & 842.87 & 8.65 & 14.52 & 282.77 & 19.47 & 0.56 & 1.29 & 0.960 \\
\hline $645 E$ & $46 R$ & 3 & 129 & 844.39 & 7.18 & 21.00 & 463.14 & 22.06 & 0.98 & 0.29 & 0.996 \\
\hline $645 \mathrm{E}$ & $48 R$ & 1 & 134 & 860.74 & 9.00 & 8.38 & 196.26 & 23.43 & 0.39 & 0.94 & 0.957 \\
\hline $645 E$ & $48 R$ & 2 & 134 & 862.24 & 9.99 & 12.58 & 263.96 & 20.99 & 0.69 & 0.40 & 0.989 \\
\hline $645 E$ & $49 R$ & 2 & 144 & 871.54 & 22.74 & 11.21 & 266.45 & 23.76 & 1.62 & 1.36 & 0.962 \\
\hline $645 \mathrm{E}$ & $49 R$ & 5 & 135 & 875.95 & 7.15 & 1.76 & 4595.00 & 2615.20 & 39.59 & 43.15 & 0.984 \\
\hline $645 E$ & $50 R$ & 2 & 145 & 881.15 & 21.19 & 30.83 & 259.38 & 8.41 & 1.31 & 1.75 & 0.943 \\
\hline $645 E$ & $50 R$ & 5 & 5 & 884.25 & 20.21 & 48.36 & 5029.66 & 104.00 & 162.50 & 42.92 & 0.989 \\
\hline $645 E$ & $51 R$ & 1 & 13 & 885.03 & 21.54 & 29.39 & 2041.66 & 69.46 & 84.28 & 159.78 & 0.918 \\
\hline $645 \mathrm{E}$ & 51R & 2 & 146 & 887.86 & 4.76 & 10.56 & 439.17 & 41.60 & 0.66 & 1.64 & 0.976 \\
\hline $645 E$ & $52 R$ & 1 & 94 & 888.84 & 20.45 & 105.66 & 11683.79 & 110.58 & 444.03 & 45.60 & 0.996 \\
\hline $645 E$ & $52 R$ & 4 & 112 & 893.52 & 21.68 & 9.79 & 265.11 & 27.07 & 1.54 & 1.65 & 0.953 \\
\hline $645 E$ & $52 R$ & 5 & 57 & 894.47 & 21.43 & 9.97 & 237.48 & 23.83 & 1.21 & 0.70 & 0.975 \\
\hline $645 E$ & $53 R$ & 1 & 30 & 897.90 & 24.27 & 8.85 & 243.75 & 27.54 & 1.43 & 1.65 & 0.944 \\
\hline $645 E$ & $53 R$ & 3 & 130 & 901.90 & 14.61 & 9.80 & 267.16 & 27.25 & 0.83 & 0.95 & 0.966 \\
\hline $645 E$ & $53 R$ & 5 & 135 & 904.95 & 23.92 & 11.40 & 267.27 & 23.45 & 1.37 & 1.68 & 0.941 \\
\hline
\end{tabular}


Appendix (continued)

\begin{tabular}{|c|c|c|c|c|c|c|c|c|c|c|c|}
\hline Hole & Core & & $\begin{array}{l}\text { Depth } \\
(\mathrm{cm})\end{array}$ & $\begin{array}{l}\text { SBD } \\
\text { (mbsf) }\end{array}$ & $\begin{array}{l}\text { MASS } \\
(\mathrm{g})\end{array}$ & $\underset{10-6 \mathrm{~m}^{3} / \mathrm{kg}}{\mathrm{X}}$ & $\begin{array}{c}\mathrm{X}_{\mathrm{ARM}} \\
10-6 \mathrm{~m}^{3} / \mathrm{kg}\end{array}$ & $\mathrm{X}_{\mathrm{ARM}} / \mathrm{X}$ & $\underset{(\mathrm{mA} / \mathrm{m} \mathrm{kg})}{\mathrm{SIRM}}$ & $\underset{(\mathrm{mA} / \mathrm{m} \mathrm{kg})}{\operatorname{HIRM}}$ & $\mathrm{S}$ \\
\hline $645 \mathrm{E}$ & $54 \mathrm{R}$ & 1 & 133 & 908.53 & 14.75 & 86.36 & 10201.43 & 118.12 & 211.24 & 120.01 & 0.983 \\
\hline $645 \mathrm{E}$ & $54 \mathrm{R}$ & 2 & 141 & 910.11 & 20.80 & 48.56 & 5274.45 & 108.62 & 174.54 & 34.98 & 0.992 \\
\hline $645 E$ & $54 R$ & 3 & 147 & 911.67 & 18.53 & 10.71 & 275.39 & 25.71 & 1.16 & 2.23 & 0.929 \\
\hline $645 E$ & $54 \mathrm{R}$ & 4 & 145 & 913.15 & 19.71 & 8.41 & 177.00 & 21.04 & 0.78 & 1.10 & 0.944 \\
\hline $645 \mathrm{E}$ & $55 R$ & 2 & 83 & 919.13 & 18.45 & 10.62 & 359.11 & 33.81 & 3.92 & 0.65 & 0.994 \\
\hline $645 \mathrm{E}$ & $55 R$ & 3 & 103 & 920.83 & 17.25 & 17.70 & 804.38 & 45.45 & 21.83 & 127.94 & 0.798 \\
\hline $645 \mathrm{E}$ & $55 R$ & 6 & 89 & 925.19 & 25.01 & 11.25 & 170.84 & 15.18 & 0.97 & 0.62 & 0.968 \\
\hline $645 E$ & $56 R$ & 1 & 121 & 927.81 & 15.20 & 9.92 & 164.23 & 16.56 & 0.63 & 0.72 & 0.965 \\
\hline $645 E$ & $56 \mathrm{R}$ & 3 & 109 & 930.69 & 7.10 & 115.01 & 6275.04 & 54.56 & 62.63 & 38.51 & 0.991 \\
\hline $645 E$ & $56 R$ & 5 & 140 & 934.00 & 19.32 & 10.73 & 201.97 & 18.82 & 0.86 & 0.83 & 0.963 \\
\hline $645 E$ & $56 R$ & 6 & 8 & 934.18 & 23.92 & 9.72 & 192.54 & 19.82 & 0.99 & 0.54 & 0.974 \\
\hline $645 E$ & $57 R$ & 1 & 49 & 936.69 & 23.39 & 10.90 & 254.80 & 23.37 & 1.41 & 1.56 & 0.949 \\
\hline $645 E$ & $57 R$ & 2 & 78 & 938.48 & 20.21 & 11.44 & 252.06 & 22.04 & 1.30 & 1.71 & 0.947 \\
\hline $645 \mathrm{E}$ & $57 R$ & 3 & 93 & 940.13 & 17.99 & 9.57 & 233.45 & 24.40 & 1.06 & 1.73 & 0.941 \\
\hline $645 \mathrm{E}$ & $57 R$ & 4 & 12 & 940.82 & 19.78 & 9.97 & 266.25 & 26.70 & 1.25 & 2.34 & 0.926 \\
\hline $645 \mathrm{E}$ & $57 R$ & 5 & 122 & 943.42 & 16.21 & 9.61 & 208.09 & 21.65 & 0.84 & 0.71 & 0.973 \\
\hline $645 E$ & $57 R$ & 6 & 87 & 944.57 & 21.99 & 9.37 & 219.55 & 23.43 & 1.14 & 0.33 & 0.987 \\
\hline $645 \mathrm{E}$ & $58 R$ & 1 & 16 & 946.06 & 13.45 & 33.35 & 3301.64 & 99.01 & 53.35 & 77.89 & 0.961 \\
\hline $645 E$ & $58 R$ & 3 & 146 & 950.36 & 12.32 & 10.81 & 253.50 & 23.45 & 0.65 & 1.45 & 0.945 \\
\hline $645 E$ & $58 R$ & 4 & 116 & 951.56 & 20.59 & 10.56 & 230.51 & 21.84 & 1.06 & 0.30 & 0.988 \\
\hline $645 \mathrm{E}$ & $58 R$ & 5 & 134 & 953.24 & 21.25 & 9.70 & 238.60 & 24.61 & 1.16 & 0.85 & 0.969 \\
\hline $645 \mathrm{E}$ & $59 R$ & 1 & 124 & 956.84 & 17.43 & 10.88 & 205.63 & 18.89 & 0.77 & 0.11 & 0.995 \\
\hline $645 \mathrm{E}$ & $59 R$ & 2 & 115 & 958.25 & 19.33 & 9.16 & 237.80 & 25.95 & 1.03 & 1.41 & 0.947 \\
\hline $645 \mathrm{E}$ & $59 R$ & 3 & 130 & 959.90 & 18.84 & 9.60 & 217.72 & 22.67 & 0.97 & 1.33 & 0.948 \\
\hline $645 E$ & $59 R$ & 4 & 88 & 960.98 & 17.87 & 9.77 & 228.48 & 23.38 & 0.96 & 1.97 & 0.927 \\
\hline $645 \mathrm{E}$ & $59 R$ & 5 & 93 & 962.53 & 19.08 & 9.74 & 184.03 & 18.89 & 0.69 & 0.23 & 0.987 \\
\hline $645 E$ & $59 R$ & 6 & 97 & 964.07 & 19.12 & 9.72 & 174.97 & 17.99 & 0.73 & 0.86 & 0.955 \\
\hline $645 E$ & $60 R$ & 1 & 54 & 965.84 & 16.40 & 10.42 & 205.83 & 19.76 & 0.73 & 1.10 & 0.950 \\
\hline $645 \mathrm{E}$ & $60 R$ & 4 & 36 & 970.16 & 18.35 & 10.41 & 226.68 & 21.78 & 0.90 & 1.12 & 0.954 \\
\hline $645 \mathrm{E}$ & 60R & 5 & 34 & 971.64 & 18.35 & 9.58 & 166.84 & 17.41 & 0.65 & 0.19 & 0.989 \\
\hline $645 E$ & 61R & 1 & 108 & 975.98 & 17.89 & 20.43 & 1676.00 & 82.02 & 34.93 & 16.32 & 0.983 \\
\hline $645 \mathrm{E}$ & 61R & 3 & 104 & 978.94 & 19.12 & 12.29 & 197.64 & 16.09 & 0.77 & 0.68 & 0.966 \\
\hline $645 E$ & 61R & 5 & 25 & 981.15 & 21.14 & 9.15 & 214.29 & 23.42 & 0.85 & 0.81 & 0.960 \\
\hline $645 E$ & 61R & 6 & 2 & 982.42 & 20.62 & 18.64 & 200.63 & 10.76 & 0.75 & 0.95 & 0.948 \\
\hline $645 E$ & $62 R$ & 1 & 125 & 985.75 & 13.67 & 8.45 & 208.15 & 24.62 & 0.71 & 1.00 & 0.962 \\
\hline $645 \mathrm{E}$ & $62 R$ & 2 & 83 & 986.83 & 12.63 & 10.64 & 227.38 & 21.36 & 0.54 & 1.42 & 0.934 \\
\hline $645 E$ & $62 R$ & 3 & 122 & 988.72 & 18.90 & 10.83 & 199.14 & 18.38 & 0.73 & 1.02 & 0.947 \\
\hline $645 E$ & $62 R$ & 4 & 112 & 990.12 & 17.77 & 10.68 & 230.19 & 21.56 & 0.98 & 0.77 & 0.972 \\
\hline $645 \mathrm{E}$ & $62 R$ & 5 & 112 & 991.62 & 20.50 & 11.70 & 253.09 & 21.62 & 1.29 & 1.05 & 0.967 \\
\hline $645 E$ & $62 R$ & 6 & 51 & 992.51 & 15.69 & 10.65 & 206.82 & 19.42 & 0.68 & 0.66 & 0.969 \\
\hline $645 E$ & $63 R$ & 1 & 142 & 995.62 & 16.37 & 10.67 & 236.98 & 22.22 & 0.88 & 0.81 & 0.970 \\
\hline $645 E$ & $63 R$ & 2 & 142 & 997.12 & 19.37 & 9.86 & 211.76 & 21.48 & 0.67 & 0.27 & 0.984 \\
\hline $645 E$ & $63 R$ & 3 & 142 & 998.62 & 19.62 & 9.35 & 201.25 & 21.53 & 0.59 & 0.84 & 0.944 \\
\hline $645 \mathrm{E}$ & $63 R$ & 4 & 142 & 1000.12 & 22.81 & 8.48 & 192.77 & 22.73 & 0.85 & 0.97 & 0.948 \\
\hline $645 E$ & $63 R$ & 5 & 2 & 1000.22 & 17.25 & 9.10 & 200.28 & 22.00 & 0.63 & 1.32 & 0.927 \\
\hline $645 E$ & $63 R$ & 6 & 131 & 1003.01 & 18.62 & 9.24 & 172.25 & 18.64 & 0.57 & 0.19 & 0.987 \\
\hline $645 E$ & $64 R$ & 1 & 140 & 1005.30 & 17.02 & 10.26 & 204.90 & 19.97 & 0.56 & 0.54 & 0.967 \\
\hline $645 E$ & $65 R$ & 2 & 140 & 1016.50 & 17.96 & 10.56 & 215.86 & 20.44 & 0.82 & 4.59 & 0.800 \\
\hline $645 \mathrm{E}$ & $65 R$ & 3 & 141 & 1018.01 & 12.94 & 11.07 & 186.11 & 16.82 & 0.51 & 0.15 & 0.992 \\
\hline $645 \mathrm{E}$ & $65 R$ & 4 & 140 & 1019.50 & 18.34 & 9.45 & 158.37 & 16.75 & 0.67 & 0.21 & 0.989 \\
\hline $\begin{array}{l}645 E \\
645 E\end{array}$ & $\begin{array}{l}65 R \\
65 R\end{array}$ & $\begin{array}{l}5 \\
7\end{array}$ & $\begin{array}{l}40 \\
40\end{array}$ & $\begin{array}{l}1020.00 \\
1023.00\end{array}$ & $\begin{array}{l}19.16 \\
17.54\end{array}$ & $\begin{array}{r}9.90 \\
11.67\end{array}$ & $\begin{array}{l}162.41 \\
250.75\end{array}$ & $\begin{array}{l}16.40 \\
21.48\end{array}$ & $\begin{array}{l}0.60 \\
1.16\end{array}$ & $\begin{array}{l}0.46 \\
3.32\end{array}$ & $\begin{array}{l}0.970 \\
0.900\end{array}$ \\
\hline $645 E$ & $66 R$ & 4 & 117 & 1028.97 & 18.11 & 8.74 & 231.49 & 26.48 & 0.63 & 0.77 & 0.956 \\
\hline $645 E$ & $67 R$ & 3 & 141 & 1037.31 & 15.48 & 10.06 & 198.91 & 19.77 & 0.52 & 0.92 & 0.945 \\
\hline $645 E$ & $68 R$ & 5 & 23 & 1048.83 & 14.60 & 9.29 & 188.27 & 20.26 & 0.36 & 0.52 & 0.958 \\
\hline $645 E$ & $69 R$ & 2 & 125 & 1054.95 & 20.33 & 10.20 & 230.00 & 22.56 & 0.60 & 0.14 & 0.991 \\
\hline $645 \mathrm{E}$ & $69 R$ & 6 & 108 & 1060.78 & 20.82 & 21.54 & 227.18 & 10.55 & 0.51 & 2.06 & 0.833 \\
\hline $645 E$ & $71 R$ & 2 & 126 & 1073.86 & 17.81 & 7.69 & 243.92 & 31.72 & 0.51 & 0.71 & 0.951 \\
\hline
\end{tabular}


Appendix (continued)

\begin{tabular}{|c|c|c|c|c|c|c|c|c|c|c|}
\hline Hole & Core S & $\begin{array}{l}\text { Depth } \\
\text { (cm) }\end{array}$ & $\begin{array}{c}\text { SBD } \\
\text { (mbsf) }\end{array}$ & $\begin{array}{l}\text { MASS } \\
(\mathrm{g})\end{array}$ & $\underset{10^{-6} \mathrm{~m}^{3} / \mathrm{kg}}{X}$ & $\underset{10-6 \mathrm{~m}^{3} / \mathrm{kg}}{\mathrm{X}_{\mathrm{ARM}}}$ & $\mathrm{X}_{\mathrm{ARM}} / \mathrm{X}$ & $\underset{(\mathrm{mA} / \mathrm{m} \mathrm{kg})}{\operatorname{SIRM}}$ & $\underset{(\mathrm{mA} / \mathrm{m} \mathrm{kg})}{\operatorname{HIRM}}$ & S \\
\hline $645 \mathrm{E}$ & $72 \mathrm{R} 2$ & $2 \quad 57$ & 1082.77 & 19.60 & 8.72 & 314.78 & 36.11 & 0.71 & 1.26 & 0.931 \\
\hline $645 E$ & $73 R 2$ & 2131 & 1093.21 & 21.89 & 10.22 & 313.12 & 30.65 & 0.73 & 0.67 & 0.960 \\
\hline $645 \mathrm{E}$ & $73 R 5$ & 577 & 1097.17 & 22.88 & 9.88 & 273.16 & 27.64 & 1.04 & 0.63 & 0.972 \\
\hline $645 \mathrm{E}$ & $74 \mathrm{R} 6$ & 694 & 1108.24 & 24.07 & 10.28 & 284.82 & 27.70 & 1.00 & 0.31 & 0.985 \\
\hline $645 E$ & $75 \mathrm{R} 1$ & 1136 & 1110.66 & 16.23 & 10.76 & 306.06 & 28.45 & 0.73 & 0.64 & 0.971 \\
\hline $645 \mathrm{E}$ & $76 R 2$ & 2143 & 1121.73 & 16.23 & 8.67 & 252.57 & 29.13 & 1.20 & 2.04 & 0.945 \\
\hline $645 \mathrm{E}$ & $78 R 3$ & 3136 & 1142.06 & 19.16 & 11.67 & 256.96 & 22.02 & 0.94 & 0.87 & 0.965 \\
\hline
\end{tabular}

